\title{
Yeast Nuak1 phosphorylates histone H3 threonine 11 in low glucose stress conditions by the cooperation of AMPK and CK2 signaling
}

Seunghee $\mathrm{Oh}^{1}$, Jaehyoun Lee ${ }^{1}$, Selene K. Swanson ${ }^{1}$, Laurence Florens ${ }^{1}$, Michael P. Washburn ${ }^{1,2}$, and Jerry L. Workman ${ }^{1} *$

${ }^{1}$ Stowers Institute for Medical Research, 1000 E. $50{ }^{\text {th }}$ Street, Kansas City, MO 64110, USA

${ }^{2}$ Department of Pathology and Laboratory Medicine, University of Kansas Medical Center, Kansas City, KS 66160, USA

*Corresponding author

Email: jlw@stowers.org 


\begin{abstract}
Changes in available nutrients are inevitable events for most living organisms. Upon nutritional stress, several signaling pathways cooperate to change the transcription program through chromatin regulation to rewire cellular metabolism. In budding yeast, histone H3 threonine 11 phosphorylation (H3pT11) acts as a marker of low glucose stress and regulates the transcription of nutritional stress responsive genes. Understanding how this histone modification 'senses' external glucose changes remains elusive. Here, we show that Tda1, the yeast orthologue of human Nuak1, is a direct kinase for H3pT11 upon low glucose stress. Yeast AMPK directly phosphorylates Tda1 to govern Tda1 activity, while CK2 regulates Tda1 nuclear localization. Collectively, AMPK and CK2 signaling converge on histone kinase Tda1 to link external low glucose stress to chromatin regulation.
\end{abstract}




\section{Introduction}

To ensure survival, cells must properly adapt to changes in available nutrients by altering their metabolism. This adaptation can be achieved through the cooperation of multiple metabolic pathways. Among those pathways, AMPK (AMP-activated protein kinase) signaling has a central role in energy homeostasis, especially when the cellular nutrient supply is low (González, Hall, Lin, \& Hardie, 2020). AMPK orthologues are very well-conserved from yeast to human, and occur as heterotrimeric complex comprising catalytic $\alpha$ subunit and regulatory $\beta$ and $\gamma$ subunits (Ross, MacKintosh, \& Hardie, 2016). For its proper function, the AMPK catalytic $\alpha$ subunit needs to be phosphorylated at its conserved threonine residue (threonine 172 in rat (Hawley et al., 1996), and threonine 210 in budding yeast (McCartney \& Schmidt, 2001)) within the activation loop of its kinase domain. In budding yeast, the catalytic $\alpha$ subunit is Snf1 (Sucrose Non-Fermenting 1). This name comes from its requirement for growth by sucrose fermentation (Carlson, Osmond, \& Botstein, 1981). The regulatory $\gamma$ subunit of budding yeast AMPK is Snf4, and its interaction with Snf1 liberates Snf1 from auto-inhibition that interferes with Snf1 threonine 210 (T210) phosphorylation (Chen et al., 2009). Budding yeast encodes three AMPK $\beta$ subunit proteins; Sip1, Sip2 and Ga183. They are partially redundant. Only when all three $\beta$ subunits are deleted, cells exhibit growth defects in media containing ethanol or glycerol as a sole carbon source, as the snfl $\Delta$ mutant does (Erickson \& Johnston, 1993; Schmidt $\&$ McCartney, 2000). The $\beta$ subunits have a conserved $C$ terminal sequence that interacts with $\alpha$ and $\gamma$ subunits (Jiang \& Carlson, 1997; X. Yang, Jiang, \& Carlson, 1994) and a specific N terminal sequence for each $\beta$ subunit which confers a distinctive subcellular localization of the Snf1 complex . Notably, Gal83 is required for Snf1 nuclear localization upon glucose depletion (Olivier Vincent, Townley, Kuchin, \& Carlson, 2001). 
Snf1 interacts with and phosphorylates several proteins involved in nutritional stress responses (Coccetti, Nicastro, \& Tripodi, 2018). Its substrates include several transcription factors such as Cat8 and Sip4 which bind to Carbon Source Responsive Elements (CSRE) for the transcription of gluconeogenic genes (Lesage, Yang, \& Carlson, 1996; Randez-Gil, Bojunga, Proft, \& Entian, 1997; O. Vincent \& Carlson, 1998). Cat8 is required for proper expression of the transcription factor Adr1, which is also phosphorylated by Snf1 (Kacherovsky, Tachibana, Amos, Fox, \& Young, 2008; Young, Kacherovsky, \& Van Riper, 2002). Snf1 also phosphorylates the Mig1 transcription repressor, which suppresses the transcription of glucose repressive genes (Nehlin \& Ronne, 1990). Mig1 phosphorylation by Snf1 activates its nuclear export signal leading to expulsion of Mig1 from the nucleus (DeVit \& Johnston, 1999; Papamichos-Chronakis, Gligoris, \& Tzamarias, 2004; Treitel, Kuchin, \& Carlson, 1998). Interestingly, the yeast hexokinase $2(\mathrm{Hxk} 2)$ protein interacts with Mig1 in the nucleus to protect Mig1 from phosphorylation by Snf1 (Ahuatzi, Riera, Pelaez, Herrero, \& Moreno, 2007). When glucose becomes scarce, Hxk2 is phosphorylated at serine 15 which inactivates its nuclear localization signal and, in turn, facilitates its cytoplasmic localization (Fernandez-Garcia, Pelaez, Herrero, \& Moreno, 2012; Kriegel, Rush, Vojtek, Clifton, \& Fraenkel, 1994). Snf1 had been believed to be a Hxk2 serine 15 kinase, however, recent studies revealed that yeast Nuak1 homolog Tda1 is responsible for Hxk2 phosphorylation (Kaps et al., 2015; Kettner et al., 2012). Thus far, Hxk2 is the only known substrate of Tda1, and how Tda1 activity is regulated upon low glucose stress is unknown.

AMPK also has a central role in regulating nutritional stress-specific chromatin modification (Lee, Oh, Abmayr, \& Workman, 2020). In yeast, Snf1 phosphorylates H3 at serine 10 at the INOl gene promoter to regulate its transcription (Lo et al., 2001). Human AMPK 
phosphorylates $\mathrm{H} 2 \mathrm{~B}$ at serine 36 at the promoters of $\mathrm{p} 53$ responsive genes upon glucose starvation (Bungard et al., 2010). AMPK indirectly regulates H3 arginine 17 di-methylation by affecting histone arginine methyltransferase CARM1 protein level in the nucleus upon apoptosisinducing conditions (Shin et al., 2016).

Recent studies imply that metabolic enzymes themselves also participate in chromatin regulation. Pyruvate kinase, a glycolysis enzyme, can phosphorylate $\mathrm{H} 3$ at threonine 11 in humans and in yeast (Li et al., 2015; W. Yang et al., 2012). Especially in yeast, pyruvate kinase forms a complex named SESAME with several metabolic enzymes involved in serine and SAM metabolism to regulate $\mathrm{H} 3$ threonine 11 phosphorylation (H3pT11) in glucose rich conditions (Li et al., 2015). Interestingly, we found that H3pT11 can act as a marker of low glucose stress in yeast (Oh, Suganuma, Gogol, \& Workman, 2018). The global H3pT11 level is inversely correlated with external glucose level and specifically increased at genes involved in metabolic changes. Notably, the H3pT11 increase upon low glucose stress is a SESAME-independent process, indicating multiple different kinases are involved in $\mathrm{H} 3 \mathrm{pT} 11$ regulation. We found that Cka1, a catalytic subunit of CK2 complex, is required for H3pT11 regulation in low glucose conditions. However, CK2 has been observed to be constitutively active and insensitive to external changes of environmental cues (Pinna, 2002). It remains elusive how a constantly active CK2 can regulate H3pT11, which becomes elevated upon low glucose stress. This suggests that H3pT11 may require additional mechanisms for sensing the availability of carbon sources.

In this study, we identify an understudied kinase, Tda1, as a histone H3 T11 kinase in low glucose stress conditions. Snf1 directly phosphorylates Tda1 at its C-terminus, especially at tandem serine 483 and threonine 484 (S483/T484) residues, which is required for in vivo Tda1 activity. CK2 regulates H3pT11 by controlling Tda1 nuclear localization. Hence, Tda1 acts as a 
signaling platform that can combine Snf1 and CK2 signaling, thereby connecting external nutrient availability to transcription regulation in the nucleus.

\section{Results}

\section{H3pT11 phosphorylation upon low glucose stress is dependent on Snf1.}

We previously reported a genome-wide study that histone $\mathrm{H} 3$ threonine 11 phosphorylation (H3pT11) specifically increases at the promoters of stress responsive genes upon low glucose stress, and H3pT11 is required for proper transcription of those genes (Oh et al., 2018). As AMPK signaling has major regulatory roles in nutrient starvation conditions (González et al., 2020), we tested whether H3pT11 is linked with the yeast AMPK homolog, Snf1 signaling pathway. Upon the media shift from glucose rich (2\%) YPD to nutritionally unfavorable YP with 3\% glycerol (YPgly), Snf1 threonine 210 phosphorylation (Snf1 pT210), the active Snf1 marker, showed a similar pattern of increase compared to that of H3pT11 (Figure 1A). In addition, a snf1 $\triangle$ mutant showed significantly reduced global H3pT11 levels in saturated cultures (Figure 1B) where media glucose is depleted (Oh et al., 2018), and impaired H3pT11 increase upon media shift from YPD to YPgly (Figure 1C). Rim15 is another kinase which becomes active in low glucose conditions (Vidan \& Mitchell, 1997; Wei et al., 2008), but a rim15 mutant did not show the global H3pT11 defect (Figure 1B). Snf1 phosphorylates several transcription factors including Adr1, Cat8, Sip4 and Mig1 upon nutritional stress (Kacherovsky et al., 2008; Lesage et al., 1996; Randez-Gil et al., 1997; Treitel et al., 1998), however, deletion of these transcription factors did not affect H3pT11 levels (Figure 1-figure supplement 1). These results suggest that $\mathrm{H} 3 \mathrm{pT} 11$ is specifically regulated via the Snf1 pathway and reduction of H3pT11 in a snf1 $\triangle$ mutant is not an indirect result of a transcriptional defect. Previously, we 
showed that Cka1, the catalytic subunit of CK2, is required for the H3pT11 increase in low glucose stress conditions (Oh et al., 2018). Interestingly, a cka1 $\Delta$ snfl $\Delta$ double deletion mutant showed a similar level of H3pT11 compared to a snf1 $\triangle$ or ckal $\Delta$ single deletion mutant (Figure 1D), suggesting that CK2 and Snf1 may act in the same pathway for H3pT11 regulation.

Snf1 can phosphorylate histone $\mathrm{H} 3$ at serine 10 in yeast (Lo et al., 2001). As H3 serine 10 is next to $\mathrm{H} 3 \mathrm{~T} 11$, we tested whether histone $\mathrm{H} 3 \mathrm{~S} 10$ phosphorylation (H3pS10) and H3pT11 behave similarly. Upon media shift from YPD to YPgly, the global H3pS10 level remained stable, while H3pT11 was increased (Figure 1E). In addition, an H3S10A mutant, where histone H3 serine 10 is mutated into alanine, showed an unperturbed H3pT11 increase upon glucose depletion (Figure 1F). These results indicate that $\mathrm{H} 3 \mathrm{pT} 11$ and $\mathrm{H} 3 \mathrm{pS} 10$ are differently regulated under low glucose stress, and Snf1 signaling, but not H3pS10, is required for H3pT11 increase in the stress condition.

\section{Snf1 and CK2 are not direct kinases for H3pT11}

Snf1 forms a functional AMPK complex with the regulatory $\beta$ and $\gamma$ subunits (Ross et al., 2016). When we measured H3pT11 levels from Snf1 complex subunit deletion mutants, interestingly, only the catalytic $\alpha$ subunit mutant, snf1 1 , showed a defect while $\beta$ (Gal83, Sip1, and Sip2) and $\gamma$ subunit (Snf4) deletion mutants showed a comparable level of H3pT11 to WT (Figure 2A). Indeed, a $\beta$ subunit mutant (gal834) showed a slightly increased level of H3pT11. As Gal83 dictates Snf1 nuclear localization upon low glucose stress (Olivier Vincent et al., 2001), this result raised the possibility that Snf1 may not be a direct kinase or a major kinase for H3pT11 in vivo.

Our previous study showed that yeast TAP purified CK2 can phosphorylate H3 at T11 in 
vitro (Oh et al., 2018). However, recombinant yeast Cka1 and recombinant human CK2 complex did not phosphorylate $\mathrm{H} 3$ at T11 in vitro, while both TAP purified and recombinant CK2 phosphorylated $\mathrm{H} 3$ at S10 (Figures 2B and 2C). CK2 is unusual for a kinase in that it can utilize both ATP and GTP as phosphate donors (Niefind, Pütter, Guerra, Issinger, \& Schomburg, 1999). When GTP was used as the phosphate donor, TAP purified CK2 phosphorylated H3 at S10, but not at T11 (Figure 2D). In addition, the CK2 specific inhibitor CX-4945 (Siddiqui-Jain et al., 2010) suppressed CK2 activity against H3pS10, but not against H3pT11 in vitro (Figure 2E). These results indicate that an unknown CK2 interacting kinase, rather than CK2 itself, is responsible for $\mathrm{H} 3 \mathrm{pT} 11$ in vitro.

\section{Tda1 is responsible for $\mathrm{H3pT11}$ upon low glucose stress in vivo}

Since Snf1 and CK2 are not direct kinases against H3pT11, we inquired as to which kinase might be responsible for the modification. As the Snf1 pathway is critical in nutritional stress conditions, we hypothesized that Snf1 interacting kinases may be responsible or participate in H3pT11 regulation. Using the Saccharomyces Genome Database (https://www.yeastgenome.org) as a guide, we tested H3pT11 levels of deletion mutants of the kinases which are known to interact with the Snf1 complex. Unexpectedly, the yeast Nuak1 homolog tdal $\triangle$ mutant showed a significantly reduced H3pT11 levels (Figure 3A). In glucose depleted media, the tdal $\Delta$ mutant showed a more severe defect in the global H3pT11 levels than the snf1 1 mutant (Figure 3B). H3pS10 levels did not significantly change in tda1 $\triangle$, supporting the independence of $\mathrm{H} 3 \mathrm{pT} 11$ from $\mathrm{H} 3 \mathrm{pS} 10$ in this stress condition. In media-shift assays, the tdal $\Delta$ mutant showed impaired H3pT11 increase in YPgly, similar to snfl $($ Figure 3C). Interestingly, upon media shift from YPD to YPgly, Tda1 protein levels were significantly 
increased in both the cytoplasm and the nucleus in the wildtype strain (Figure 3D and Figure 3figure supplement 1). Collectively, these results strongly suggest that Tda1 has a central role in H3pT11 regulation upon low glucose stress.

\section{Tda1 phosphorylates $\mathrm{H3}$ at $\mathrm{T11}$ in vitro}

Thus far, the only known Tda1 target is yeast hexokinase2 (Hxk2) at serine 15 (Kaps et al., 2015; Kettner et al., 2012). We noticed that the surrounding sequence of Hxk2 serine 15 is similar to that of histone H3 T11 (Figure 4A). To test if Tda1 can directly phosphorylate H3 in vitro, we purified Tda1 from yeast using TAP tag purification (Figure 4B). TAP purified Tda1 did not show many bands in a PAGE gel, suggesting that Tda1 may not be a part of multisubunit complex. An in vitro assay showed that indeed, TAP purified Tda1 protein can robustly phosphorylate H3 at T11 (Figure 4C). Recombinant GST tagged Tda1 purified from E. coli also phosphorylated H3 at T11 but not at S10, while Snf1 purified from yeast using a FLAG tag phosphorylated H3 at both S10 and T11 in vitro (Figure 4D). Sequence analysis from Saccharomyces Genome Database (http//www.yeastgenome.org) predicts that Tda1 may contain a kinase domain at the N-terminus. In this regard, we made several Tda1 fragments (Tda1 N1 N5) to define the kinase domain (Figure 4E). An in vitro kinase assay of the fragments showed that Tda1 N1 (amino acids 1 to 353) did not phosphorylate H3 at T11 unlike Tda1 N2 (amino acids 1 to 380). Interestingly, Tda1 N3 (amino acids 1 to 426) showed a reduced activity against H3pT11 compared to Tda1 N2 (Figure 4F). These results suggest that Tda1 catalytic domain resides in amino acid 1 to 380, and the amino acids patch spanning 381 to 426 has a potential inhibitory effect on Tda1 activity against H3pT11. 


\section{Snf1 directly phosphorylates Tda1 at C-terminus}

Next, we inquired how Snf1 signaling can regulate the activity of Tda1 towards H3pT11. irst, we compared the changes in Tda1 protein levels upon media shift from YPD to YPgly in WT and snf1 (Figure 5A). Interestingly, Tda1 showed multiple species migrating differently on a PAGE gel. In a low glucose stress condition, slowly migrating Tda1 species became dominant in the WT strain. However, in the snfl $\Delta$ mutant, the faster migrating species was dominant. In the ckal $\triangle$ mutant, Tda1 protein level changes were similar to that of WT (Figure 5-figure supplement 1A). We speculated that the apparent size differences of Tda1 in WT and snflA reflected differential post translational modification patterns of Tda1. To test this possibility, Tda1 was purified from yeast using FLAG tag, then incubated with $\lambda$ phosphatase. After the phosphatase treatment, Tda1 showed a more distinctive one band pattern while the slower migrating bands disappeared, indicating Tda1 is indeed a phosphorylated protein (Figure 5B). In addition, when we compared Tda1 purified from WT, snfl 1 , and ckal $\Delta$ backgrounds, the phosphorylated species of Tda1 was significantly reduced specifically in the snfl $\triangle$ mutant, implying that Snf1 is responsible for Tda1 phosphorylation (Figure 5C).

Tda1 co-immunoprecipitated with Snf1 as well as with Cka1 in vivo (Figure 5-figure supplement 1B and 1C). In this regard, we tested whether Snf1 can directly phosphorylate Tda1 in vitro. Snf1 was purified from a reg1 $\triangle$ strain using FLAG tag, maintaining a robust Snf1 pT210 signal (Figure 5-figure supplement 1D), as Reg1 recruits the Snf1 pT210 phosphatase Glc7 and inactivates Snf1 (Tu \& Carlson, 1995). Then the purified Snf1-FLAG was incubated with GST-tagged Tda1 fragments, Tda1 N1 (amino acids 1 to 353) or Tda1C (aa 354 to 586) (Figure 5D). Interestingly, Snf1 robustly phosphorylated only the Tda1C fragment (Figure 5E). The pattern of protein band migration in the PAGE gel also indicated phosphorylated Tda1C. An 
in vitro assay with a recombinant Snf1 kinase domain (aa 41-315, Snf1-KD) also showed that Snf1 can phosphorylate Tda1C fragment (Figure 5F).

\section{Tda1 phosphorylation by Snf1 is required for Tda1 activity}

To investigate which residues of Tda1 are modified by Snf1, the Tda1C fragment was incubated with recombinant Snf1-KD or recombinant GST-Cka1 and then subjected to MudPIT (Multi-dimensional protein identification technology) mass spectrometry analysis. Surprisingly, MudPIT analysis revealed that Snf1 phosphorylates multiple serine and threonine residues of the Tda1C fragment (Figure 6A and Supplemental Table S1). We categorized Tda1 phosphorylation sites by Snf1 into three groups by their proximity to each other. Group I includes multiple serine and threonine residues residing in amino acids from 396 to 417. Interestingly, this region is located just after the Tda1 kinase domain. Group II includes tandem serine 483 and threonine 484. These two residues showed the highest PTM percentages among tested residues. Group III includes serine 570. MudPIT analysis also revealed that Cka1 robustly phosphorylated Tda1C at serine 578 .

To understand how Tda1 phosphorylation by Snf1 or Cka1 regulates its function, we generated Tda1 phosphorylation site defective mutants and tested their H3pT11 levels. Strikingly, among the tested mutants, H3pT11 level was significantly reduced only in the group II site defective mutant, Tda1 S483A/T484A (Figure 6B). Tda1 S483A/T484A mutant also showed impaired H3pT11 increase, while Tda1 $\Delta 396-417$ mutant showed intact H3pT11 increase upon media shift from YPD to YPgly (Figure 6C). Interestingly, Both Tda1 S483A/T484A and $\Delta 396-$ 417 mutants showed the robust increase of Tda1 phosphorylation upon media shift (Figure 6C Tda1-FLAG panel), which was significantly reduced in snfl $\triangle$ background (Figure 5A). These 
results suggest that $\mathrm{Snf1}$ is responsible for the multi-sites phosphorylation of Tda1 and among those phosphorylation, S483/T484 phosphorylation has a critical role for H3pT11 regulation.

We inquired how Tda1 S483/T484 phosphorylation can regulate the function of Tda1 for H3pT11. First, we hypothesized that the availability of Tda1 for H3pT11 may be affected by its phosphorylation. However, Tda1 S483A/T484A mutant showed a similar level of nuclear Tda1 compared to Tda1 WT (Figure 6-figure supplement 1A and 1B), suggesting that Tda1 S483/T484 phosphorylation is not required for Tda1 nuclear localization. Next, we hypothesized that Tda1 S483/T484 phosphorylation may regulate Tda1 activity against H3T11. To test this hypothesis, we purified Tda1 WT or Tda1 S483A/T484A protein from yeast for in vitro kinase assays. Interestingly, the Tda1 S483A/T484A mutant showed significantly lower activity against H3 T11 than Tda1 WT (Figure 6D). These results indicate that Snf1 regulates Tda1 activity by phosphorylating Tda1 S483/T484 residues.

\section{CK2 regulates Tda1 nuclear localization}

Both Snf1 and CK2 are required for proper H3pT11 levels in low glucose stress conditions, and they genetically interact with each other in H3pT11 regulation (Figure 1D). CK2 does not affect global Tda1 phosphorylation levels (Figure 5C). Tda1 purified from cka1 $\Delta$ background showed similar activity against H3 T11 to Tda1 purified from WT (Figure 7-figure supplement 1), suggesting that CK2 does not affect Tda1 activity. To investigate how CK2 regulates Tda1 function, we tested Tda1 subcellular localization in WT and ckal $\Delta$ mutant. Interestingly, we found that Tda1 nuclear localization was significantly decreased in $c k a 1 \triangle$ mutants in low glucose stress conditions compared to WT (Figure 7A). As we could not find any conventional NLS sequence in Tda1, we attached a strong cMyc nuclear localization signal 
(PAAKRVKLD) (Dang \& Lee, 1988) at the C-terminal end of Tda1 to see if the cMyc NLS could help Tda1 to bypass the requirement for Cka1 for its nuclear localization. The DNA construct expressing Tda1 tagged with C-terminal 3xFLAG (Tda1 WT) or 3xFLAG with cMyc NLS (Tda1 cNLS) under a strong ADH1 promoter was integrated into the genome of a tdal $\triangle$ mutant. Upon low glucose stress, Tda1 cNLS showed a slightly lower level of the protein increase than Tda1 WT. However, the Tda1 cNLS expressing strain showed a more robust and rapid increase of H3pT11 compared to the Tda1 WT expressing strain (Figure 7B). Next, we integrated the Tda1 WT or Tda1 cNLS construct into tda1 $\Delta c k a 1 \Delta$ or $t d a 1 \Delta s n f 1 \Delta$ backgrounds. Interestingly, in the ckal $\triangle$ mutant, the Tda1 cNLS was able to mediate H3pT11, unlike Tda1 WT (Figure 7C, left panel). On the contrary, neither Tda1 cNLS nor Tda1 WT could restore H3pT11 in snf1 1 mutant (Figure 7C, right panel). Thus, while Snf1 regulates Tda1 activity Cka1 regulates its nuclear localization. These results clearly show that Snf1 and Cka1 signaling pathways cooperatively regulate $\mathrm{H} 3 \mathrm{pT} 11$ but use different mechanisms or act in different stages of the Tda1 regulation (Summarized in Figure 7D).

\section{Discussion}

\section{Tda1 phosphorylation by Snf1}

In vitro kinase assays using recombinant Tda1 fragments and Snf1 revealed multiple Tda1 residues phosphorylated by Snf1 (Figure 6A and Supplemental Table S1). Among Tda1 phosphorylation site-defective mutants tested, only a Tda1 S483A/T484A mutant showed a significant defect in $\mathrm{H} 3 \mathrm{pT} 11$ regulation in vivo (Figures $6 \mathrm{~B}$ and $\mathrm{C}$ ), suggesting the importance of Tda1 S483/T484 phosphorylation. Indeed, the Tda1 S483/T484 residues showed the highest level of modification in the in vitro kinase assay (Supplemental Table S1). 
Tda1 S483/T484 phosphorylation is required for Tda1 kinase activity on H3 T11 (Figure 6D), however, How the modification regulates Tda1 activity remains unclear. Tda1 S483/T484 residues are not located in the Tda1 catalytic domain (Figure 4E, Tda1 aa 1-380). Tda1 S483/T484 phosphorylation may regulate Tda1 activity by relieving an unknown inhibition mechanism, or Tda1 may require additional factors to become fully active, and Tda1 phosphorylation at S483/T484 may be responsible for interacting with such factors. Investigating Tda1 binding proteins which depend on Tda1 phosphorylation would be an exciting future step.

\section{Cka1 regulates Tda1 nuclear localization}

Both Snf1 and Cka1 are required for $\mathrm{H} 3 \mathrm{pT} 11$ regulation, but two kinases regulate H3pT11 via different mechanisms. Snf1 robustly phosphorylates the Tda1 C terminal (Figures 5E and F), while Cka1 does not affect global Tda1 phosphorylation (Figure 5C). A Tda1 S578A point mutation at the putative phosphorylation site by Cka1 does not affect the global level of H3pT11 (Figure 6B). Tda1 with cMyc NLS behaves differently in the snflA and ckal $\triangle$ backgrounds (Figure 7C). The NLS-attached Tda1 can bypass the requirement for Cka1, but not that for Snf1. This result suggests that Cka1 regulates the nuclear localization process of Tda1 by controlling the interaction between Tda1 and nuclear importins, in contrast to Snf1 which regulates Tda1 enzymatic activity (Figure 6D). Tda1 does not possess conventional phosphorylation NLS sequence in it. In this regard, it is not clear if Tda1 itself contains nonconventional NLS, or Tda1 requires other accessory factors for its nuclear localization. Finding a Tda1 specific importin would also be an important next step. 
CK2 has been known as a constitutively active kinase complex, which does not require upstream stimulation for its activity. However, many processes governed by CK2 are highly controlled processes (Pinna, 2002). In this study, we show an example of how a constitutively active kinase can be involved in tightly regulated processes such as H3pT11 upon low glucose stress. Although CK2 is constitutively active, H3pT11 cannot be achieved without active Snf1. Previously, we reported that Sch9 was also required for $\mathrm{H} 3 \mathrm{pT} 11$ regulation as well as $\mathrm{CK} 2$ (Oh et al., 2018). Interestingly, Sch9 and CK2 genetically interact in H3pT11 regulation as Cka1 and Snf1 do. Our finding of H3pT11 regulation by CK2 via controlling the Tda1 nuclear localization suggests a possibility that $\mathrm{Sch} 9$ is also involved in the same process. This raises the possibility of Tda1 being a signaling platform that combines multiple signaling such as Snf1, CK2, and Sch9 together to finely connect external glucose levels to chromatin.

\section{Tda1 in higher eukaryotes}

Structural analysis (at http://yeastgenome.org) predicts that Tda1 contains a calcium/calmodulin dependent kinase domain at its N-terminus. We found that its functional kinase domain resides between amino acid 1 to 380 (Figure 4F). In yeast, there are 12 kinases that contain the domain including yeast calcium calmodulin dependent kinases (Cmk1 and Cmk2) and meiosis specific kinase Mek1, as well as Tda1. Interestingly, Mek1 phosphorylates H3 at T11 during meiosis (Kniewel et al., 2017). A large scale screening of yeast phosphorylation site motifs revealed that the amino acid sequence surrounding H3T11 fits in the recognition motif of Cmk1 and Cmk2 (Mok et al., 2010). These results imply that calcium/calmodulin dependent kinases could be kinase candidates for H3pT11 in situations other than nutrient starvation in yeast and possibly in other species. 
Based on predictions by the PPOD program (at http://ppod.princeton.edu/), Nuak1 has been proposed as a human ortholog of yeast Tda1 (Soma, Yang, Morales, \& Polymenis, 2014). Nuak1 is also known as ARK5 which means AMPK related kinase 5. As its name suggests, Nuak1 has a 47\% amino acid sequence homology to AMPK $\alpha 1$ (Suzuki, Kusakai, Kishimoto, Lu, Ogura, Lavin, et al., 2003). Nuak1 is involved in cell survival processes upon nutrient starvation (Suzuki, Kusakai, Kishimoto, Lu, Ogura, \& Esumi, 2003), suggesting the Tda1 function upon nutrient stress shown in yeast is consistent in higher eukaryotes. Nuak1 predominantly localizes to the nucleus, but a recent study revealed that the cellular distribution of Nuak1 changes upon stresses in a importin $\beta$-dependent manner (Palma et al., 2019). Investigating whether the Nuak1 subcellular localization is regulated via a similar mechanism to that of Tda1 and phosphorylates histones under a specific stress like Tda1 would be an interesting future study.

\section{Materials and Methods}

\section{Yeast strains and culture conditions}

All yeast strains used in this study are listed in Table S2. All single deletion mutants using KanMX4 cassette and TAP tagged strains derived from BY4741 were obtained from Open Biosystems library (maintained at the Stowers Institute Molecular Biology facility). Yeast synthetic histone H3 mutants (Dai et al., 2008) (H3 WT, H3 T11A and H3 S10A in Figure 1F) were also purchased from Open Biosystems. Other deletions and tagged strains were made by targeted homologous recombination of PCR fragments containing marker genes flanked by gene specific sequences. These strains were confirmed by PCR with primer sets specific for their marker genes. For Tda1 mutant strains shown in Figures 6C, 6D and 7, Tda1 WT or Tda1 mutant constructs were cloned into pRS406 vector and the DNA constructs were linearized by NcoI 
digestion then integrated into URA3 loci of the genome. For low glucose stress experiments, yeast cells were saturated by overnight culture at $30^{\circ} \mathrm{C}$. For media shift experiments, the saturated cultures were inoculated into fresh YPD media and then incubated until mid-log phase (Optical density 0.4-0.6). These cultures were washed once with YPgly (YP with 3\% glycerol) media, then resuspended with YPgly for indicated times at $30^{\circ} \mathrm{C}$.

\section{Preparation of yeast whole cell extracts}

Yeast whole cell extracts were prepared as previously described (Oh et al., 2018). 5 OD cells were taken from the cultures. Cell pellets were washed once with distilled water, then resuspended in $250 \mathrm{uL}$ of $2 \mathrm{M} \mathrm{NaOH}$ with $8 \% \beta$-Mercaptoethanol for 5 minutes on ice. Cells were pelleted then washed once with $250 \mathrm{uL}$ TAP extraction buffer (40 mM HEPES pH 7.5, 10\% Glycerol, $350 \mathrm{mM} \mathrm{NaCl}, 0.1 \%$ Tween-20, phosphatase inhibitor cocktail and proteinase inhibitor cocktail from Roche). Cell pellets were resuspended in $180 \mathrm{uL}$ modified 2X SDS buffer then boiled at $100{ }^{\circ} \mathrm{C}$ for $4-5$ minutes. For detecting Snf1 T210 phosphorylation (Figure 1A), cell cultures were boiled for 5 minutes before initial harvesting to prevent Snf1 phosphorylation by centrifugation (Orlova, Barrett, \& Kuchin, 2008).

\section{Yeast flag tagged protein purification}

Tda1 and Snf1 proteins with 3xFLAG tag were purified as previously described (Dutta et al., 2014) with minor modifications. 6L cultures of cells were grown in YPD to $2-3 \mathrm{OD}$ at $30^{\circ} \mathrm{C}$ and collected. For Tda1-3xFLAG purification, cell pellets were resuspended in 3L YP without glucose and then incubated for an additional 1 hour. Cell pellets were washed once with distilled water, then resuspended in buffer A (25 mM HEPES pH 7.5, 10\% glycerol, $350 \mathrm{mM} \mathrm{KCl,} 2 \mathrm{mM}$ 
$\mathrm{MgCl}_{2}, 1 \mathrm{mM}$ EDTA, $0.02 \%$ NP40, supplemented with $20 \mu \mathrm{g} / \mathrm{mL}$ leupeptin, $20 \mu \mathrm{g} / \mathrm{mL}$ pepstatin and $100 \mu \mathrm{M}$ PMSF) and then broken up by bead beating. The crude cell extracts were incubated with $125 \mathrm{U}$ Benzonase and $500 \mu \mathrm{g}$ heparin for 15 minutes at RT to remove nucleic acid contamination, then the extracts were further clarified by ultracentrifugation. The clarified extracts were incubated with Anti-FLAG M2 affinity resin (Sigma) for 4 hours at $4{ }^{\circ} \mathrm{C}$ with gentle rotation. Proteins bound to the resin were washed 3 times with Buffer A, then washed once with Buffer B (25 mM HEPES pH 7.5, $10 \%$ glycerol, 100 mM KCl, 2 mM MgCl, 1 mM EDTA, $1 \mathrm{mM}$ DTT, 0.02\% NP40, supplemented with $20 \mu \mathrm{g} / \mathrm{mL}$ leupeptin, $20 \mu \mathrm{g} / \mathrm{mL}$ pepstatin and $100 \mu \mathrm{M}$ PMSF). Bound proteins were eluted in buffer B containing $0.5 \mathrm{mg} / \mathrm{mL} 3 \mathrm{xFLAG}$ peptides.

\section{Recombinant protein purification}

Yeast Cka1 and Tda1 genes were amplified by PCR from yeast genomic DNA and then cloned into pGEX4T-1 (GE healthcare) vector. GST-Snf1 kinase domain (Snf1-KD) expression vector was purchased from Addgene (\#52683). Those DNA constructs were transformed in Rosetta2 (Novagen) competent cells and protein expressions were induced by $0.5 \mathrm{mM}$ IPTG for 18 hours at $16^{\circ} \mathrm{C}$. Bacterial cell pellets were resuspended in TAP extraction buffer, then sonicated to disrupt cell walls. Crude extracts were incubated with Glutathione sepharose 4B (GE healthcare) resin for 3 hours at $4^{\circ} \mathrm{C}$. Resin bound proteins were eluted in $50 \mathrm{mM}$ Tris $\mathrm{pH} 8.0$ buffer containing $20 \mathrm{mM}$ Glutathione. Xenopus core histones were purified as previously described (Shim, Duan, Chen, Smerdon, \& Min, 2012) with minor modifications. Briefly, YS-14 construct encoding all four Xenopus core histones (H2A, H2B, H3, and H4) was transformed into Tuner DE3 pLysS competent cells (Novagen), and then histone protein expressions were 
induced in 2X YT media by $0.5 \mathrm{mM}$ IPTG for 24 hours at $37^{\circ} \mathrm{C}$. Cell pellets were sonicated in high salt extraction buffer $(20 \mathrm{mM}$ Tris $\mathrm{pH} 8.0,2 \mathrm{M} \mathrm{NaCl})$, and then clarified extracts were incubated with TALON metal affinity resin (Clontech) for 3 hours at $4^{\circ} \mathrm{C}$. Resin bound proteins were eluted by $250 \mathrm{mM}$ imidazole, then eluted proteins were dialyzed in high salt extraction buffer for overnight at $4^{\circ} \mathrm{C}$.

\section{Yeast subcellular fractionation}

Yeast cellular fractionation was carried out as previously described (Emili et al., 2006) with minor modifications. 40 to 50 OD yeast cells were pelleted, then washed successively with $10 \mathrm{~mL}$ distilled water and ice cold $10 \mathrm{~mL} \mathrm{SB}$ (1 M Sorbitol, $20 \mathrm{mM}$ Tris-Cl pH 7.5), The washed cell pellets were transferred to $1.7 \mathrm{~mL}$ Eppendorf tube, then successively washed with $1 \mathrm{~mL}$ PSB (20 mM Tris-Cl pH 7.5, 2 mM EDTA, 100 mM NaCl, 10 mM $\beta$-Mercaptoethanol) and 1mL SB. The pellets were resuspended with $750 \mu \mathrm{L} \mathrm{SB}$, then yeast cell walls were digested by adding 100 $\mu \mathrm{L}$ Zymolyase $(10 \mathrm{mg} / \mathrm{mL}$, Seikagaku) for 1 hour at RT. After the cell wall digestion, $750 \mu \mathrm{L}$ ice-cold SB was added, then the spheroplasts were collected by gentle centrifugation $(2 \mathrm{~K}, 5$ mins, $4^{\circ} \mathrm{C}$ ) and washed once with $1 \mathrm{~mL}$ ice-cold SB. The spheroplasts were resuspended by $500 \mu \mathrm{L}$ EBX (20 mM Tris-Cl pH 7.5, 100 mM NaCl, $0.25 \%$ Triton X-100, 15 mM $\beta$-Mercaptoethanol), then $100 \%$ Triton X-100 was added up to $0.5 \%$ to disrupt outer cell membrane. Cells were placed on ice for 10 minutes with occasional mixing, then $1 \mathrm{~mL}$ NIB $(20 \mathrm{mM}$ Tris-Cl pH 8.0, $100 \mathrm{mM} \mathrm{NaCl}, 1.2 \mathrm{M}$ Sucrose, $15 \mathrm{mM} \beta$-Mercaptoethanol) was layered over the cells. After high speed centrifugation $\left(12 \mathrm{~K}, 15 \mathrm{mins}, 4^{\circ} \mathrm{C}\right)$, the upper layer was taken as cytoplasmic fraction. Glassy, white nuclear pellets were resuspended SDS containing sample buffer then boiled for western blot experiments. 


\section{In vitro kinase assays}

All in vitro kinase assays were done in NEBuffer for protein kinase (NEB) at $30^{\circ} \mathrm{C}$. Recombinant Snf1 kinase domain (Snf1-KD) was phosphorylated by recombinant human CaMKK2 (Abnova) for its activation before kinase assays (Figure 5F). When Tda1 fragments were used as kinases (Figure. 4F), additional DTT was supplemented up to $1 \mathrm{mM}$. The reactions were quenched by SDS sample buffer addition, then boiled at $100^{\circ} \mathrm{C}$ for 5 minutes.

\section{Multi-dimensional Protein Identification Technology (MudPIT) analysis}

TCA-precipitated proteins were urea-denatured, reduced, alkylated and digested with endoproteinase Lys-C (Promega) followed by modified trypsin (Promega) as previously described (Florens \& Washburn, 2006; Washburn, Wolters, \& Yates, 2001). Peptide mixtures were loaded onto $100 \mu \mathrm{m}$ fused silica microcapillary columns packed with 5 - $\mu \mathrm{m} \mathrm{C}_{18}$ reverse phase (Aqua, Phenomenex), strong cation exchange particles (Partisphere SCX, Whatman), and reverse phase (MacCoss et al., 2002). Loaded microcapillary columns were placed in-line with a Quaternary Agilent 1260 series HPLC pump and a Velos Orbitrap mass spectrometer equipped with a nano-LC electrospray ionization source (ThermoFinnigan). Fully automated 10-step MudPIT runs were carried out on the electrosprayed peptides, as previously described (Florens \& Washburn, 2006). Tandem mass (MS/MS) spectra were interpreted using ProLuCID (Eng, McCormack, \& Yates, 1994) against a database of 8956 sequences, consisting of 4303 E. coli proteins (downloaded from NCBI on 2013-11-06), 177 usual contaminants such as human keratins, IgGs, and proteolytic enzymes, 4476 "shuffled" sequences and four yeast proteins sequences (Tda1, Snf1, Cka1 and a recombinant Tda1 C-terminal sequence from amino acid 
residues 354 to 586). Peptide/spectrum matches were sorted and selected using DTASelect (Tabb, McDonald, \& Yates, 2002) with the following criteria set: spectra/peptide matches were only retained if they had a DeltCn of at least 0.08, and minimum XCorr of 1.8 for singly-, 2.0 for doubly-, and 3.0 for triply-charged spectra. In addition, peptides had to be fully tryptic and at least 6 amino acids long. Combining all runs, proteins had to be detected by at least 2 such peptides, or 1 peptide with 2 independent spectra. Under these criteria, the final FDRs at all levels were zero. Peptide hits from multiple runs were compared using CONTRAST (Tabb et al., 2002). To estimate relative protein levels, distributed Normalized Spectral Abundance Factors (dNSAFs) were calculated for each detected protein, as previously described (Florens et al., 2006; Paoletti et al., 2006; Zybailov et al., 2006).

\section{Acknowledgements}

We thank Dr. Michael Church for scientific editing of the manuscript. We thank the Workman Lab members and Stowers core facilities for support during this project. These studies were supported by funds from the Stowers Institute and the National Institutes of General Medical Sciences grant R35GM1 18068 (J.L.W.). Original data underlying this manuscript can be accessed from the Stowers Original Data Repository at http://www.stowers.org/research/publications/libpb-1536.

\section{Author contributions}

S.O. and J.L.W. designed the study, analyzed the data, and wrote the manuscript. S.O. and J.L. performed the experiments. S.K.S., L.P, and M.P.W. analyzed the proteomics data. 


\section{Declaration of interests}

The authors declare no competing interest.

\section{References}

Ahuatzi, D., Riera, A., Pelaez, R., Herrero, P., \& Moreno, F. (2007). Hxk2 regulates the phosphorylation state of Mig1 and therefore its nucleocytoplasmic distribution. $J$ Biol Chem, 282(7), 4485-4493. doi:10.1074/jbc.M606854200

Bungard, D., Fuerth, B. J., Zeng, P. Y., Faubert, B., Maas, N. L., Viollet, B., . . Berger, S. L. (2010). Signaling kinase AMPK activates stress-promoted transcription via histone H2B phosphorylation. Science, 329(5996), 1201-1205. doi:10.1126/science.1191241

Carlson, M., Osmond, B. C., \& Botstein, D. (1981). Mutants of yeast defective in sucrose utilization. Genetics, 98(1), 25-40.

Chen, L., Jiao, Z.-H., Zheng, L.-S., Zhang, Y.-Y., Xie, S.-T., Wang, Z.-X., \& Wu, J.-W. (2009). Structural insight into the autoinhibition mechanism of AMP-activated protein kinase. Nature, 459(7250), 1146-1149.

Coccetti, P., Nicastro, R., \& Tripodi, F. (2018). Conventional and emerging roles of the energy sensor Snf1/AMPK in Saccharomyces cerevisiae. Microbial Cell, 5(11), 482.

Dai, J., Hyland, E. M., Yuan, D. S., Huang, H., Bader, J. S., \& Boeke, J. D. (2008). Probing nucleosome function: a highly versatile library of synthetic histone $\mathrm{H} 3$ and $\mathrm{H} 4$ mutants. Cell, 134(6), 1066-1078.

Dang, C. V., \& Lee, W. (1988). Identification of the human c-myc protein nuclear translocation signal. Molecular and cellular biology, 8(10), 4048-4054. 
DeVit, M. J., \& Johnston, M. (1999). The nuclear exportin Msn5 is required for nuclear export of the Mig1 glucose repressor of Saccharomyces cerevisiae. Curr Biol, 9(21), 1231-1241. doi:10.1016/s0960-9822(99)80503-X

Dutta, A., Gogol, M., Kim, J.-H., Smolle, M., Venkatesh, S., Gilmore, J., . . Workman, J. L. (2014). Swi/Snf dynamics on stress-responsive genes is governed by competitive bromodomain interactions. Genes \& development, 28(20), 2314-2330.

Emili, A., Kim, J. H., Onishi, M., Keogh, M.-C., Harrison, C., Haber, J. J., . . Buratowski, S. (2006). A phosphatase complex that dephosphorylates gammaH2AX regulates DNA damage checkpoint recovery. Nature.

Eng, J., McCormack, A. L., \& Yates, J. R., III. (1994). An approach to correlate tandem mass spectral data of peptides with amino acid sequences in a protein database. J. Amer. Mass Spectrom., 5, 976-989.

Erickson, J. R., \& Johnston, M. (1993). Genetic and molecular characterization of GAL83: its interaction and similarities with other genes involved in glucose repression in Saccharomyces cerevisiae. Genetics, 135(3), 655-664.

Fernandez-Garcia, P., Pelaez, R., Herrero, P., \& Moreno, F. (2012). Phosphorylation of yeast hexokinase 2 regulates its nucleocytoplasmic shuttling. J Biol Chem, 287(50), 4215142164. doi:10.1074/jbc.M112.401679

Florens, L., Carozza, M. J., Swanson, S. K., Fournier, M., Coleman, M. K., Workman, J. L., \& Washburn, M. P. (2006). Analyzing chromatin remodeling complexes using shotgun proteomics and normalized spectral abundance factors. Methods, 40(4), 303-311.

Florens, L., \& Washburn, M. P. (2006). Proteomic analysis by multidimensional protein identification technology. Methods Mol Biol, 328, 159-175. 
González, A., Hall, M. N., Lin, S.-C., \& Hardie, D. G. (2020). AMPK and TOR: The Yin and Yang of Cellular Nutrient Sensing and Growth Control. Cell Metabolism, 31(3), 472-492.

Hawley, S. A., Davison, M., Woods, A., Davies, S. P., Beri, R. K., Carling, D., \& Hardie, D. G. (1996). Characterization of the AMP-activated protein kinase kinase from rat liver and identification of threonine 172 as the major site at which it phosphorylates AMPactivated protein kinase. Journal of Biological Chemistry, 271(44), 27879-27887.

Jiang, R., \& Carlson, M. (1997). The Snf1 protein kinase and its activating subunit, Snf4, interact with distinct domains of the Sip1/Sip2/Gal83 component in the kinase complex. Molecular and cellular biology, 17(4), 2099-2106.

Kacherovsky, N., Tachibana, C., Amos, E., Fox, D., 3rd, \& Young, E. T. (2008). Promoter binding by the Adr1 transcriptional activator may be regulated by phosphorylation in the DNA-binding region. PLoS One, 3(9), e3213. doi:10.1371/journal.pone.0003213

Kaps, S., Kettner, K., Migotti, R., Kanashova, T., Krause, U., Rodel, G., . . Kriegel, T. M. (2015). Protein kinase Ymr291w/Tda1 is essential for glucose signaling in saccharomyces cerevisiae on the level of hexokinase isoenzyme ScHxk2 phosphorylation*. J Biol Chem, 290(10), 6243-6255. doi:10.1074/jbc.M114.595074

Kettner, K., Krause, U., Mosler, S., Bodenstein, C., Kriegel, T. M., \& Rodel, G. (2012). Saccharomyces cerevisiae gene YMR291W/TDA1 mediates the in vivo phosphorylation of hexokinase isoenzyme 2 at serine-15. FEBS Lett, 586(4), 455-458. doi:10.1016/j.febslet.2012.01.030

Kniewel, R., Murakami, H., Liu, Y., Ito, M., Ohta, K., Hollingsworth, N. M., \& Keeney, S. (2017). Histone $\mathrm{H} 3$ threonine 11 phosphorylation is catalyzed directly by the meiosis- 
specific kinase Mek1 and provides a molecular readout of Mek1 activity in vivo. Genetics, 207(4), 1313-1333.

Kriegel, T. M., Rush, J., Vojtek, A. B., Clifton, D., \& Fraenkel, D. G. (1994). In vivo phosphorylation site of hexokinase 2 in Saccharomyces cerevisiae. Biochemistry, 33(1), 148-152. doi:10.1021/bi00167a019

Lee, J., Oh, S., Abmayr, S. M., \& Workman, J. L. (2020). When histones are under glucose starvation. Journal of Biosciences, 45(1), 17.

Lesage, P., Yang, X., \& Carlson, M. (1996). Yeast SNF1 protein kinase interacts with SIP4, a C6 zinc cluster transcriptional activator: a new role for SNF1 in the glucose response. Mol Cell Biol, 16(5), 1921-1928. doi:10.1128/mcb.16.5.1921

Li, S., Swanson, S. K., Gogol, M., Florens, L., Washburn, M. P., Workman, J. L., \& Suganuma, T. (2015). Serine and SAM responsive complex SESAME regulates histone modification crosstalk by sensing cellular metabolism. Molecular cell, 60(3), 408-421.

Lo, W.-S., Duggan, L., Tolga, N., Belotserkovskya, R., Lane, W. S., Shiekhattar, R., \& Berger, S. L. (2001). Snf1--a histone kinase that works in concert with the histone acetyltransferase Gcn5 to regulate transcription. Science, 293(5532), 1142-1146.

MacCoss, M. J., McDonald, W. H., Saraf, A., Sadygov, R., Clark, J. M., Tasto, J. J., . . Yates, J. R., 3rd. (2002). Shotgun identification of protein modifications from protein complexes and lens tissue. Proc Natl Acad Sci U S A, 99(12), 7900-7905. Retrieved from http://www.ncbi.nlm.nih.gov/entrez/query.fcgi?cmd=Retrieve\&db=PubMed\&dopt=Citati on\&list_uids=12060738 
McCartney, R., \& Schmidt, M. (2001). Regulation of Snf1 kinase. Activation requires phosphorylation of threonine 210 by an upstream kinase as well as a distinct step mediated by the Snf4 subunit. The Journal of biological chemistry, 276(39), 36460.

Mok, J., Kim, P. M., Lam, H. Y., Piccirillo, S., Zhou, X., Jeschke, G. R., . . Jwa, M. (2010). Deciphering protein kinase specificity through large-scale analysis of yeast phosphorylation site motifs. Sci. Signal., 3(109), ra12-ra12.

Nehlin, J. O., \& Ronne, H. (1990). Yeast MIG1 repressor is related to the mammalian early growth response and Wilms' tumour finger proteins. EMBO J, 9(9), 2891-2898.

Niefind, K., Pütter, M., Guerra, B., Issinger, O.-G., \& Schomburg, D. (1999). GTP plus water mimic ATP in the active site of protein kinase CK2. Nature structural biology, 6(12), 1100-1103.

Oh, S., Suganuma, T., Gogol, M. M., \& Workman, J. L. (2018). Histone H3 threonine 11 phosphorylation by Sch9 and CK2 regulates chronological lifespan by controlling the nutritional stress response. Elife, 7. doi:10.7554/eLife.36157

Orlova, M., Barrett, L., \& Kuchin, S. (2008). Detection of endogenous Snf1 and its activation state: application to Saccharomyces and Candida species. Yeast, 25(10), 745-754.

Palma, M., Riffo, E. N., Suganuma, T., Washburn, M. P., Workman, J. L., Pincheira, R., \& Castro, A. F. (2019). Identification of a nuclear localization signal and importin beta members mediating NUAK1 nuclear import inhibited by oxidative stress. J Cell Biochem, 120(9), 16088-16107. doi:10.1002/jcb.28890

Paoletti, A. C., Parmely, T. J., Tomomori-Sato, C., Sato, S., Zhu, D., Conaway, R. C., .. . Washburn, M. P. (2006). Quantitative proteomic analysis of distinct mammalian 
Mediator complexes using normalized spectral abundance factors. Proc Natl Acad Sci U $S A, 103(50), 18928-18933$.

Papamichos-Chronakis, M., Gligoris, T., \& Tzamarias, D. (2004). The Snf1 kinase controls glucose repression in yeast by modulating interactions between the Mig1 repressor and the Cyc8-Tup1 co-repressor. EMBO reports, 5(4), 368-372.

doi:10.1038/sj.embor.7400120

Pinna, L. A. (2002). Protein kinase CK2: a challenge to canons. Journal of cell science, 115(20), 3873-3878.

Randez-Gil, F., Bojunga, N., Proft, M., \& Entian, K. D. (1997). Glucose derepression of gluconeogenic enzymes in Saccharomyces cerevisiae correlates with phosphorylation of the gene activator Cat8p. Mol Cell Biol, 17(5), 2502-2510. doi:10.1128/mcb.17.5.2502

Ross, F. A., MacKintosh, C., \& Hardie, D. G. (2016). AMP $\square$ activated protein kinase: a cellular energy sensor that comes in 12 flavours. The FEBS journal, 283(16), 2987-3001.

Schmidt, M. C., \& McCartney, R. R. (2000). $\beta \square$ subunits of Snf1 kinase are required for kinase function and substrate definition. The EMBO Journal, 19(18), 4936-4943.

Shim, Y., Duan, M.-R., Chen, X., Smerdon, M. J., \& Min, J.-H. (2012). Polycistronic coexpression and nondenaturing purification of histone octamers. Analytical biochemistry, 427(2), 190-192.

Shin, H. J., Kim, H., Oh, S., Lee, J. G., Kee, M., Ko, H. J., . . Baek, S. H. (2016). AMPKSKP2-CARM1 signalling cascade in transcriptional regulation of autophagy. Nature, 534(7608), 553-557. doi:10.1038/nature18014

Siddiqui-Jain, A., Drygin, D., Streiner, N., Chua, P., Pierre, F., O'Brien, S. E., . . Ho, C. (2010). CX-4945, an orally bioavailable selective inhibitor of protein kinase CK2, inhibits 
prosurvival and angiogenic signaling and exhibits antitumor efficacy. Cancer research, 70(24), 10288-10298.

Soma, S., Yang, K., Morales, M. I., \& Polymenis, M. (2014). Multiple metabolic requirements for size homeostasis and initiation of division in Saccharomyces cerevisiae. Microbial Cell, 1(8), 256.

Suzuki, A., Kusakai, G., Kishimoto, A., Lu, J., Ogura, T., \& Esumi, H. (2003). ARK5 suppresses the cell death induced by nutrient starvation and death receptors via inhibition of caspase 8 activation, but not by chemotherapeutic agents or UV irradiation. Oncogene, 22(40), 6177-6182. doi:10.1038/sj.onc.1206899

Suzuki, A., Kusakai, G., Kishimoto, A., Lu, J., Ogura, T., Lavin, M. F., \& Esumi, H. (2003). Identification of a novel protein kinase mediating Akt survival signaling to the ATM protein. J Biol Chem, 278(1), 48-53. doi:10.1074/jbc.M206025200

Tabb, D. L., McDonald, W. H., \& Yates, J. R., 3rd. (2002). DTASelect and Contrast: tools for assembling and comparing protein identifications from shotgun proteomics. J Proteome Res, 1(1), 21-26. Retrieved from http://www.ncbi.nlm.nih.gov/entrez/query.fcgi?cmd=Retrieve \&db=PubMed\&dopt=Citati on\&list_uids $=12643522$

Treitel, M. A., Kuchin, S., \& Carlson, M. (1998). Snf1 protein kinase regulates phosphorylation of the Mig1 repressor in Saccharomyces cerevisiae. Mol Cell Biol, 18(11), 6273-6280. doi:10.1128/mcb.18.11.6273

Tu, J., \& Carlson, M. (1995). REG1 binds to protein phosphatase type 1 and regulates glucose repression in Saccharomyces cerevisiae. The EMBO Journal, 14(23), 5939-5946. 
Vidan, S., \& Mitchell, A. P. (1997). Stimulation of yeast meiotic gene expression by the glucoserepressible protein kinase Rim15p. Mol Cell Biol, 17(5), 2688-2697. doi:10.1128/mcb.17.5.2688

Vincent, O., \& Carlson, M. (1998). Sip4, a Snf1 kinase-dependent transcriptional activator, binds to the carbon source-responsive element of gluconeogenic genes. EMBO J, 17(23), 70027008. doi:10.1093/emboj/17.23.7002

Vincent, O., Townley, R., Kuchin, S., \& Carlson, M. (2001). Subcellular localization of the Snf1 kinase is regulated by specific $\beta$ subunits and a novel glucose signaling mechanism. Genes \& development, 15(9), 1104-1114.

Washburn, M. P., Wolters, D., \& Yates, J. R., 3rd. (2001). Large-scale analysis of the yeast proteome by multidimensional protein identification technology. Nat Biotechnol, 19(3), 242-247. Retrieved from http://www.ncbi.nlm.nih.gov/entrez/query.fcgi?cmd=Retrieve \&db=PubMed\&dopt=Citati on\&list_uids $=11231557$

Wei, M., Fabrizio, P., Hu, J., Ge, H., Cheng, C., Li, L., \& Longo, V. D. (2008). Life span extension by calorie restriction depends on Rim15 and transcription factors downstream of Ras/PKA, Tor, and Sch9. PLoS Genet, 4(1), e13. doi:10.1371/journal.pgen.0040013

Yang, W., Xia, Y., Hawke, D., Li, X., Liang, J., Xing, D., . . Lu, Z. (2012). PKM2 phosphorylates histone $\mathrm{H} 3$ and promotes gene transcription and tumorigenesis. Cell, 150(4), 685-696. doi:10.1016/j.cell.2012.07.018

Yang, X., Jiang, R., \& Carlson, M. (1994). A family of proteins containing a conserved domain that mediates interaction with the yeast $\mathrm{SNF} 1$ protein kinase complex. The EMBO Journal, 13(24), 5878-5886. 
Young, E. T., Kacherovsky, N., \& Van Riper, K. (2002). Snf1 protein kinase regulates Adr1 binding to chromatin but not transcription activation. J Biol Chem, 277(41), 38095-38103. doi:10.1074/jbc.M206158200

Zybailov, B., Mosley, A. L., Sardiu, M. E., Coleman, M. K., Florens, L., \& Washburn, M. P. (2006). Statistical analysis of membrane proteome expression changes in Saccharomyces cerevisiae. J Proteome Res, 5(9), 2339-2347.

\section{Figure legends}

\section{Figure 1. H3pT11 is an AMPK dependent, but H3pS10 independent histone modification} upon low glucose stress. (A) Comparison of the Snf1 threonine 210 phosphorylation (Snf1 pT210) and H3pT11 levels upon media shift from YPD (2\% glucose) to YPgly (YP with 3\% glycerol) measured by western blots. (B) Global H3pT11 levels in WT (BY4741), cka1A, rim15A, and snf1 $\Delta$ cells measured by western blot. The cells were taken from overnight saturated cultures in YPD media. (C) (Upper panel) Comparison of H3pT11 levels in WT and snf1 4 upon the media shift from YPD to YPgly at indicated time points analyzed by western blots. (Lower panel) Relative ratios of $\mathrm{H} 3 \mathrm{pT} 11$ to $\mathrm{H} 3$ signals presented with error bars indicating standard deviations (STD) of three biological replicates. (D) (Upper panel) Global H3pT11 levels in WT, cka1 $1, \operatorname{snfl} 1$, and ckal $\Delta s n f 1 \Delta$ cells taken from saturated cultures in YPD media measured by western blots. (Lower panel) Relative band intensities of H3pT11 to H3 signals. Error bars indicate STD from three biological replicates. (E) Changes in H3pT11 and H3pS10 signals in the WT strain (BY4741) upon the media shift from YPD to YPgly at indicated time points measured by western blots. (F) Comparison of H3pT11 upon media shift from YPD to YPgly in WT (y1166), H3T11A, and H3S10A strains analyzed by western blots. 


\section{Figure 1-figure supplement 1. H3pT11 depends on Snf, but not on Snf1 target}

transcription factors. Global H3pT11 levels in WT (BY4741), snf1A, and the deletion mutants of Snf1 target transcription factors (adr1 , cat8 8 , sip $4 \Delta$ and mig 14 ) analyzed by western blots. The cells were taken from overnight saturated cultures in YPD media.

Figure 2. Snf1 and CK2 are not direct kinases for H3pT11. (A) (Left) Global H3pT11 and H3pS10 levels of Snf1 complex $\alpha$ subunit (Snf1), $\gamma$ subunit (Snf4), and $\beta$ subunit (Gal83, Sip1, and Sip2) deletion mutants compared to WT (BY4741) analyzed by western blots. Cells were taken from saturated cultures in YPD media. (Right) The relative band intensities of H3pT11 to H3 with error bars indicating STD of three biological replicates. (B) In vitro kinase assay of recombinant GST-Cka1 and yeast TAP purified CK2 (yCK2) using recombinant Xenopus histone $\mathrm{H} 3$ as a substrate. (C) In vitro kinase assay of human recombinant CK2 complex (rCK2) and $\mathrm{yCK} 2$ using recombinant $\mathrm{H} 3$ as a substrate. (D) In vitro kinase assay of yCK2 with recombinant $\mathrm{H} 3$ as a substrate and ATP or GTP as a phosphate donor. (E) In vitro kinase assay of yCK2 for $\mathrm{H} 3$ with increasing amount of CX-4945 treatment.

Figure 3. Tda1 is responsible for H3pT11 upon low glucose stress in vivo. (A) Global H3pT11 levels in Snf1-interacting kinase mutant cells taken from saturated cultures in YPD media measured by western blots. (B and C) (Left panels) H3pT11 levels in WT, snf1 1 , and tdal $\Delta$ cells taken from (B) saturated cultures in YPD media or taken at (C) indicated time points upon media shift from YPD to YPgly analyzed by western blots. (Right panels) The relative 
band intensities of $\mathrm{H} 3 \mathrm{pT} 11$ to $\mathrm{H} 3$ are presented with error bars indicating STD of three biological replicates. (D) Tda1 protein levels tagged with C-terminal 3xFLAG tag in the nuclei, cytoplasm, and whole cell extracts (WCE) upon media shift from YPD to YPgly media at indicated time points measured by western blots against FLAG tag.

\section{Figure 3-figure supplement 1. Confirmation of efficient yeast subcellular fractionation of}

the samples shown in Figure 3D. $1 \%$ of whole cell extract (WCE), $1 \%$ of cytoplasm, and 2\% of nuclei samples used in Figure 3D were subjected to western blots to confirm efficient subcellular fractionation. Histone H3 and phosphorylated eIF2 (p-eIF2) antibodies were used for the nuclear and cytoplasmic marker, respectively.

Figure 4. Tda1 phosphorylates $\mathrm{H3}$ at T11 in vitro. (A) Comparison between the surrounding sequences of Hxk2 serine 15 and H3 (Hht1) threonine 11. (B) Silver staining of TAP purified Tda1 protein. (C) In vitro kinase assay of TAP purified Tda1 using core histones (H2A, H2B, H3, and H4) as substrates. (D) In vitro kinase assay of Snf1 complex purified from yeast using FLAG tag and recombinant yeast GST-Tda1 purified from E.coli using core histones (H2A, H2B, H3, and H4) as substrates. (E) Schematic diagram of recombinant GST-Tda1 N-terminal fragments used in (F). (F) In vitro kinase assay of Tda1 $\mathrm{N}$ fragments using core histones as substrates.

Figure 5. Snf1 phosphorylates Tda1 at C-terminus. (A) (Upper panel) Tda1-3xFLAG protein level changes in WT and snfl 1 upon media shift from YPD to YPgly measured by western blot against FLAG tag. (Lower panel) Relative band intensities of Tda1 to H3 with error bars 
indicating STD of three biological replicates. (B) Coomassie staining (upper panel) or western blot (WB) against FLAG tag (lower panel) of yeast FLAG purified Tda1 with or without $\lambda$ phosphatase treatment. (C) Coomassie staining (upper panel) or western blot against FLAG tag (lower panel) of yeast FLAG purified Tda1 in WT, snfld, and ckal $\Delta$ background. (D) Schematic diagram of Tda1 N1 (Tda1 aa 1 to 353) and Tda1 C (Tda1 aa 354 to 586) used for in vitro kinase assays shown in (E) and (F). (E) In vitro kinase assay of yeast FLAG purified Snf1 from reg1 background using GST-Tda1 N1 or GST-Tda1 C as a substrate. (F) In vitro kinase assay of recombinant Snf1 kinase domain (Snf1-KD) which was activated by human CaMKK2 using GST-Tda1 N1 or GST-Tda1 C as a substrate.

Figure 5-figure supplement 1. Snf1 phosphorylates Tda1 at $\mathbf{C}$ terminus. (A) (Left panel) Tda1-3xFLAG protein level changes in WT and cka1 $\Delta$ upon media shift from YPD to YPgly measured by western blot against FLAG tag. (Right panel) The relative band intensities of Tda1 to $\mathrm{H} 3$ with error bars indicating STD from three biological replicates. (B and C) Tda1 coimmunoprecipitation assay using (B) FLAG tagged Snf1 or (C) FLAG tagged Cka1 as a bait in YPgly media. Immunoprecipitated Tda1 was detected by western blot against HA tag. Tda1-HA strain without any FLAG tagged protein was used as a negative control. (D) The silver staining (left panel) or western blot against Snf1 pT210 (right panel) of yeast FLAG purified Snf1 from reglA background.

Figure 6. Tda1 S483/T484 phosphorylation by $\mathrm{Snf1}$ is required for the Tda1 activity in vivo. (A) Schematic map of Tda1 phosphorylation sites (denoted as red circles) by recombinant Snf1KD and Cka1. (B) Global H3pT11 levels of Tda1 phosphorylation sites defective mutants taken 
from saturated cultures in YPD media. The Tda1 constructs were expressed in pRS416 shuttling vectors and their expression was governed by ADH1 promoter. 'Vector only' construct indicates the pRS416 with ADH1 promoter only. (C) H3pT11 levels upon media shift from YPD to YPgly in $t d a 1 \Delta$ cells expressing Tda1 WT, Tda1 S483A/T484A or Tda1 $\Delta 396-417$ constructs under an ADH1 promoter. (D) In vitro kinase assay of yeast FLAG purified Tda1 WT or Tda1 S483A/T484A protein using core histones as substrates.

Figure 6-figure supplement 1. Tda1 S483/T484 phosphorylation by Snf1 is required for the nuclear Tda1 activity. (A) Subcellular localization of Tda1 WT and Tda1 S483A/T484A proteins tagged with $\mathrm{C}$ terminal 3xFLAG tag measured by western blot. The cells were taken from YPgly media cultures incubated for 2 hours after the media shift from YPD. (B) $0.5 \%$ of whole cell extract (WCE), $0.5 \%$ of cytoplasm, and $1 \%$ of nuclei samples used in (A) were subjected to western blots to confirm efficient subcellular fractionation. Histone H3 and p-eIF2 antibodies were used for the nuclear and cytoplasmic marker, respectively.

Figure 7. CK2 regulates Tda1 nuclear localization. (A) (Upper panels) Subcellular localization of Tda1-3xFLAG proteins in WT and ckal $\triangle$ mutant. WCE, cytoplasm, and nuclei samples were taken from YPgly media cultures incubated for 2 hours after the media shift from YPD, then analyzed by western blot. Histone $\mathrm{H} 3$ and p-eIF2 antibodies were used for the nuclear and cytoplasmic marker, respectively. (Lower panel) The relative band intensities of nuclear Tda1 to WCE Tda1 are presented with error bars indicating STD of three biological replicates. (B) H3pT11 level changes in Tda1 WT and Tda1 with C-terminally tagged cMyc NLS upon media shift from YPD to YPgly analyzed by western blots. (C) H3pT11 restoration by genome 
integrated Tda1 WT or Tda1 with cMyc NLS in tdal $\Delta$, tdal $\Delta c k a 1 \Delta$, and tdal $\Delta s n f 1 \Delta$

backgrounds measured by western blots. The cells were taken from YPgly media cultures

incubated for 2 hours after the media shift from YPD. (D) Summary model of the Tda1

regulation by Snf1 and Cka1. The red ellipses represent phosphorylation.

Figure 7-figure supplement 1. CK2 does not regulate Tda1 activity. In vitro kinase assay of yeast FLAG tagged Tda1 purified from WT or ckal $\Delta$ background. Core histones were used as substrates. 
A

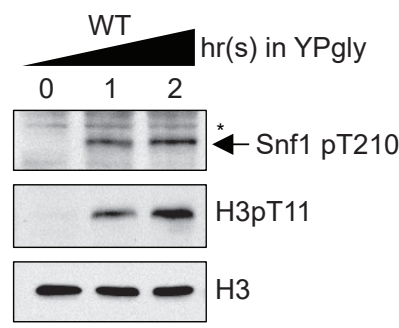

B

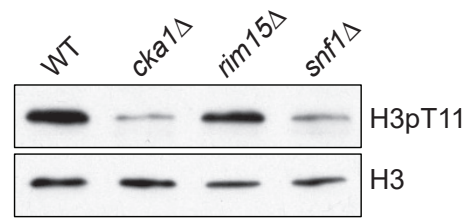

E
C
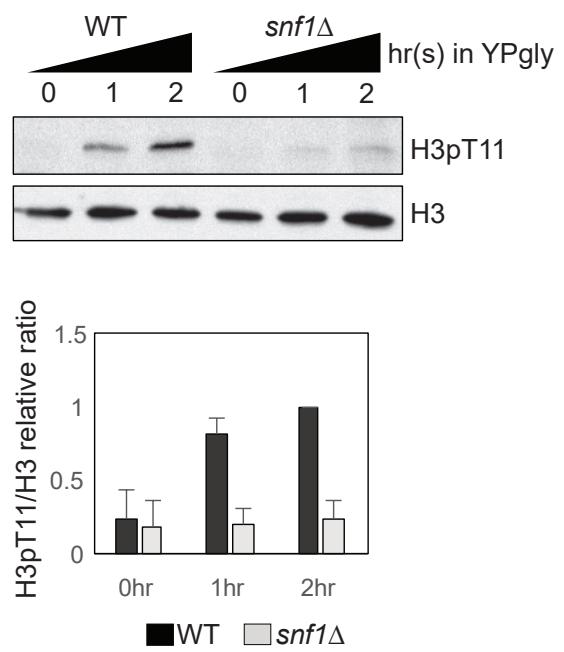

D
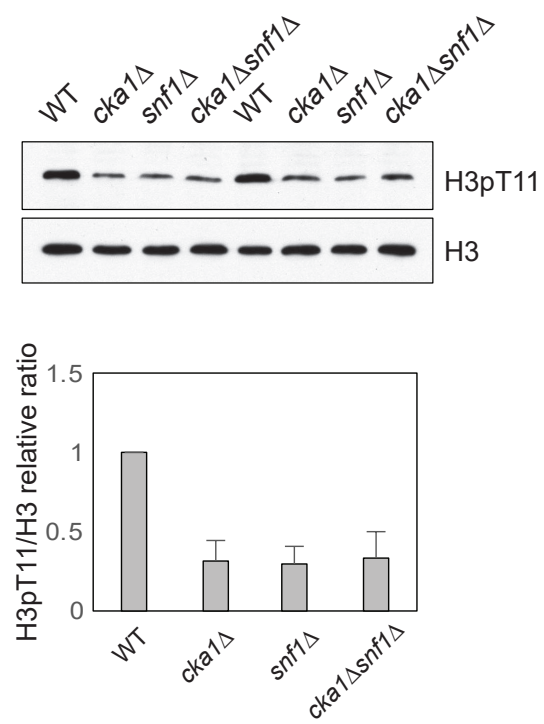

F

$\mathrm{hr}(\mathrm{s})$ in YPgly
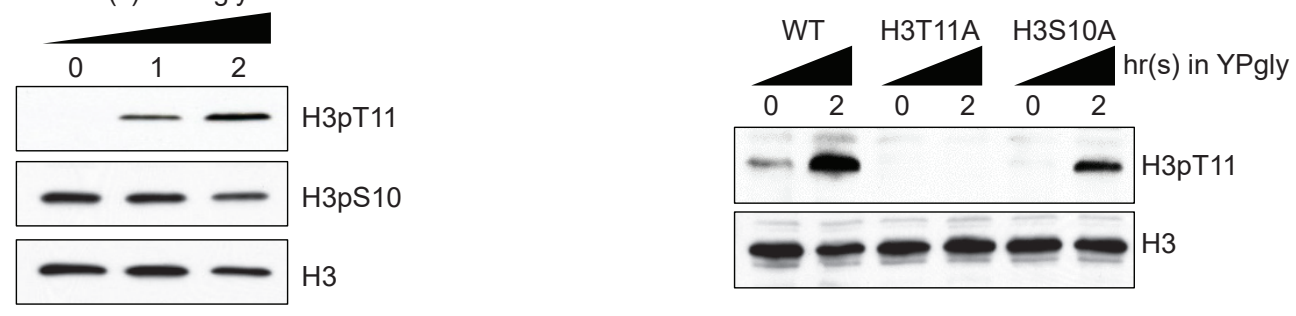
bioRxiv preprint doi: https://doi.org/10.1101/2020.11.03.367094; this version posted November 3, 2020. The copyright holder for this preprint (which was not certified by peer review) is the author/funder. All rights reserved. No reuse allowed without permission.

Figure 1-figure supplement 1

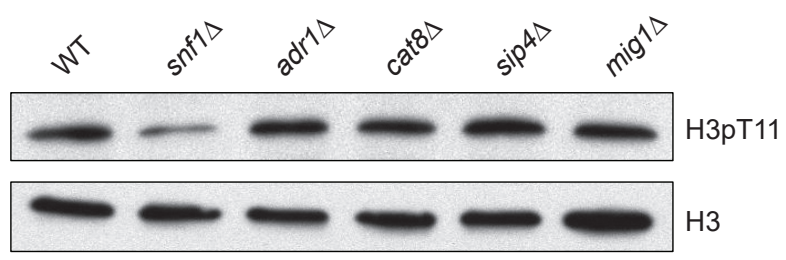


A

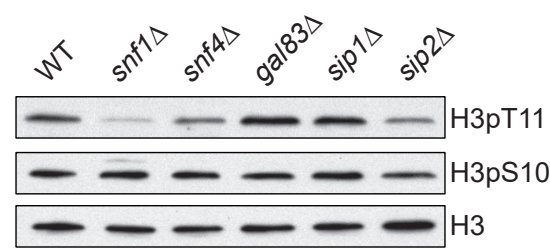

C

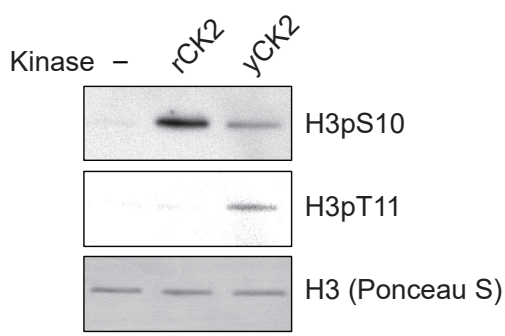

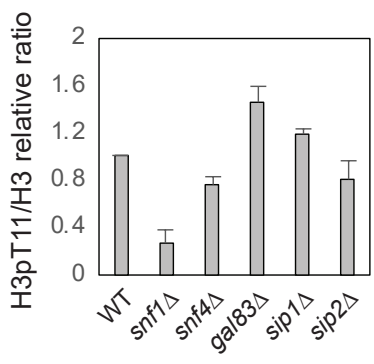

B

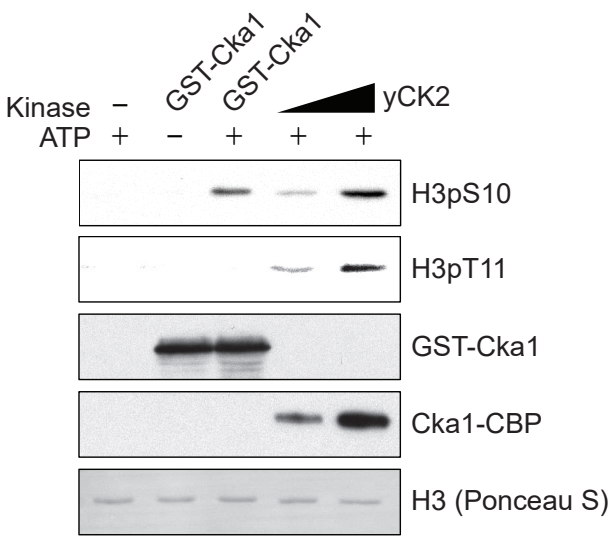

E

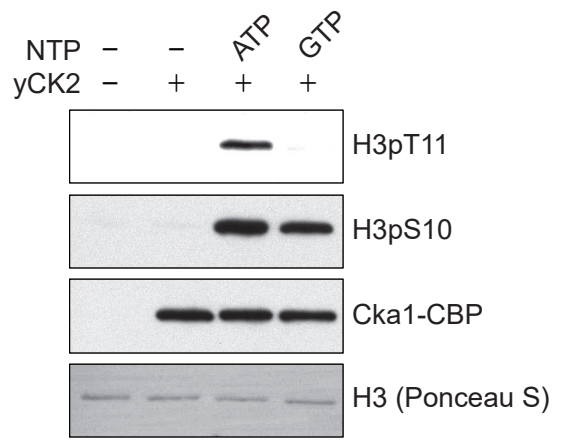

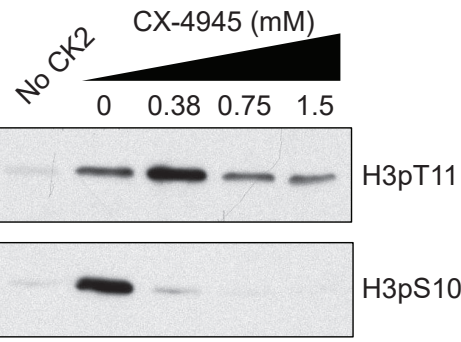

Cka1-CBP H3 (Ponceau S) 
A

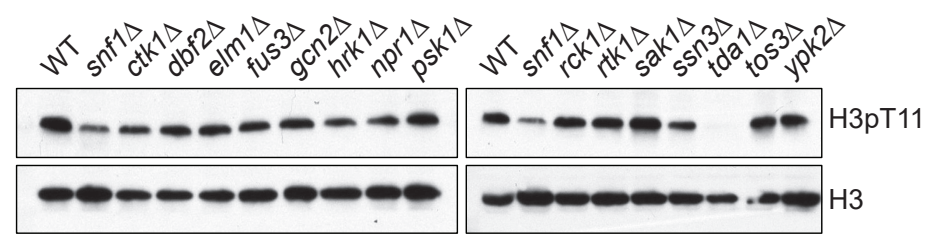

C

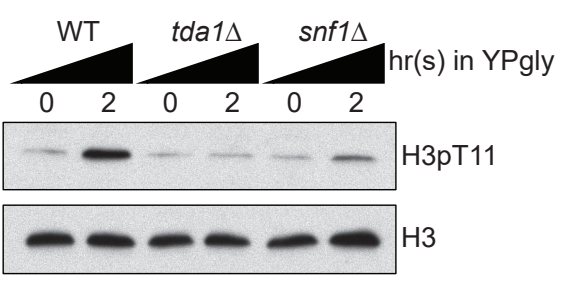

B
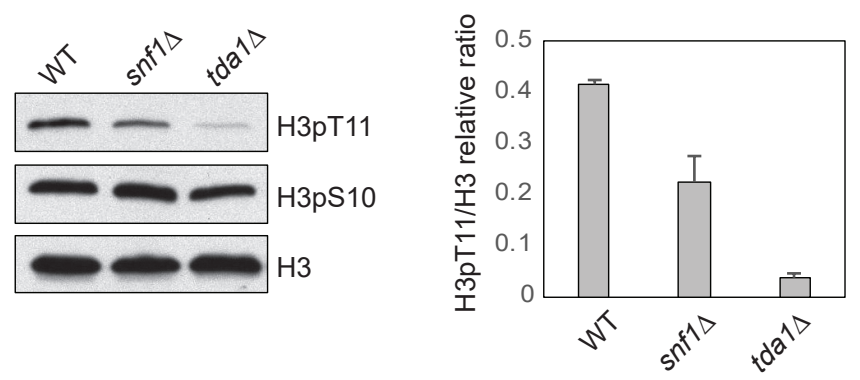

$\mathrm{D}$

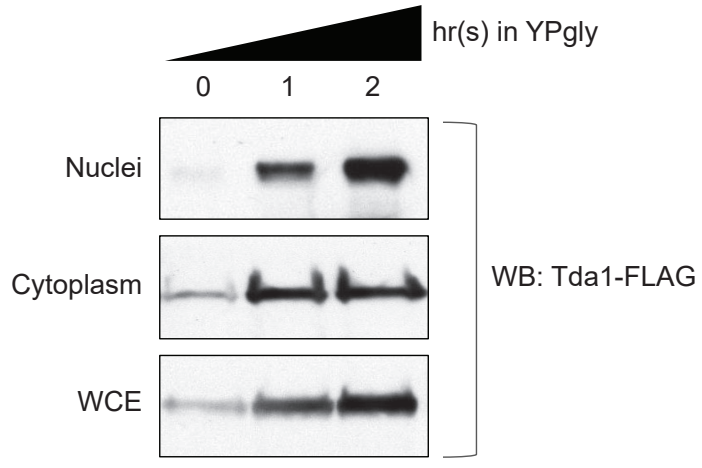


bioRxiv preprint doi: https://doi.org/10.1101/2020.11.03.367094; this version posted November 3, 2020. The copyright holder for this preprint (which was not certified by peer review) is the author/funder. All rights reserved. No reuse allowed without permission.

Figure 3-figure supplement 1

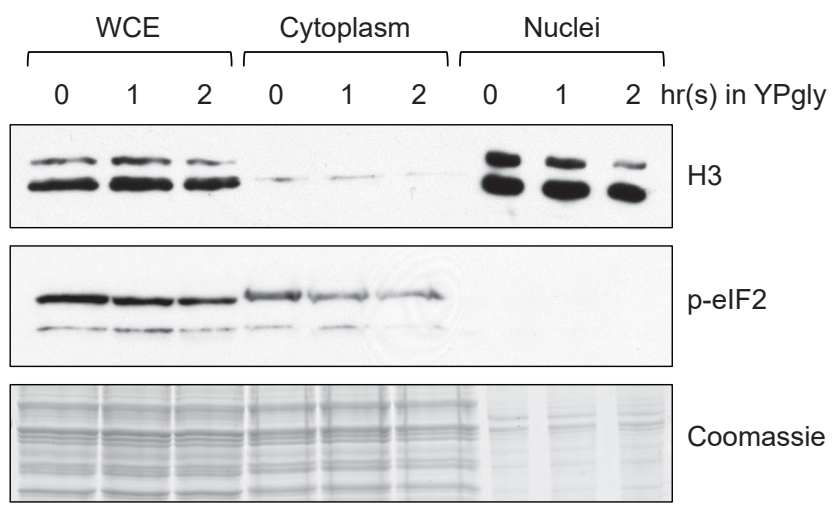


A

Hxk2 ..ARKG $\underline{S}^{15} M A D V P .$.

Hht1 ..ARKS $\overline{\mathrm{T}}^{11} \mathrm{GGKAP}$.

B

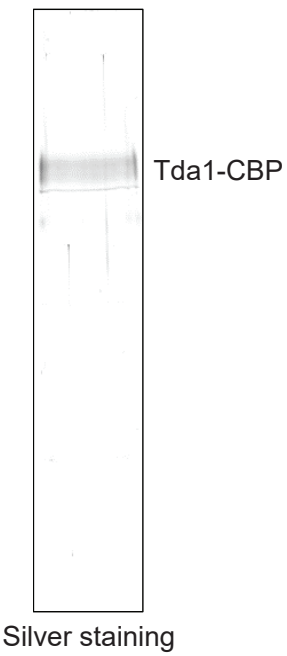

C

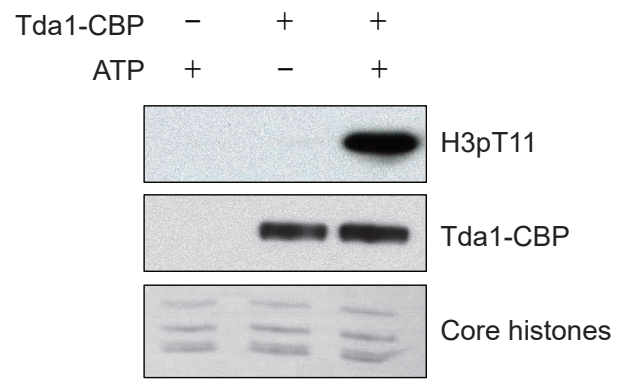

D
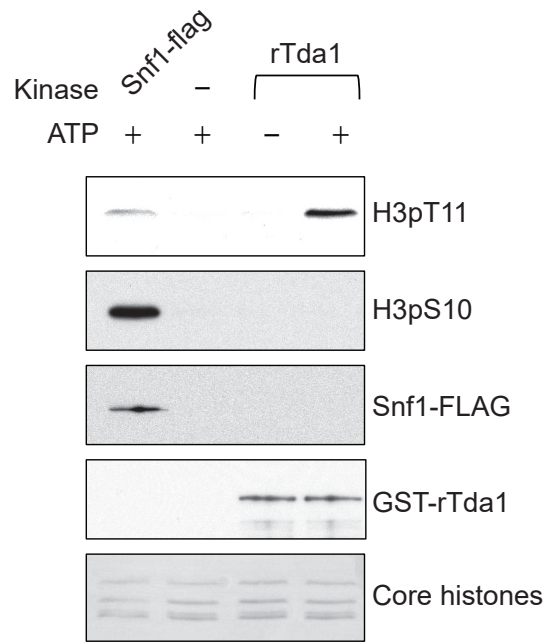

$E$

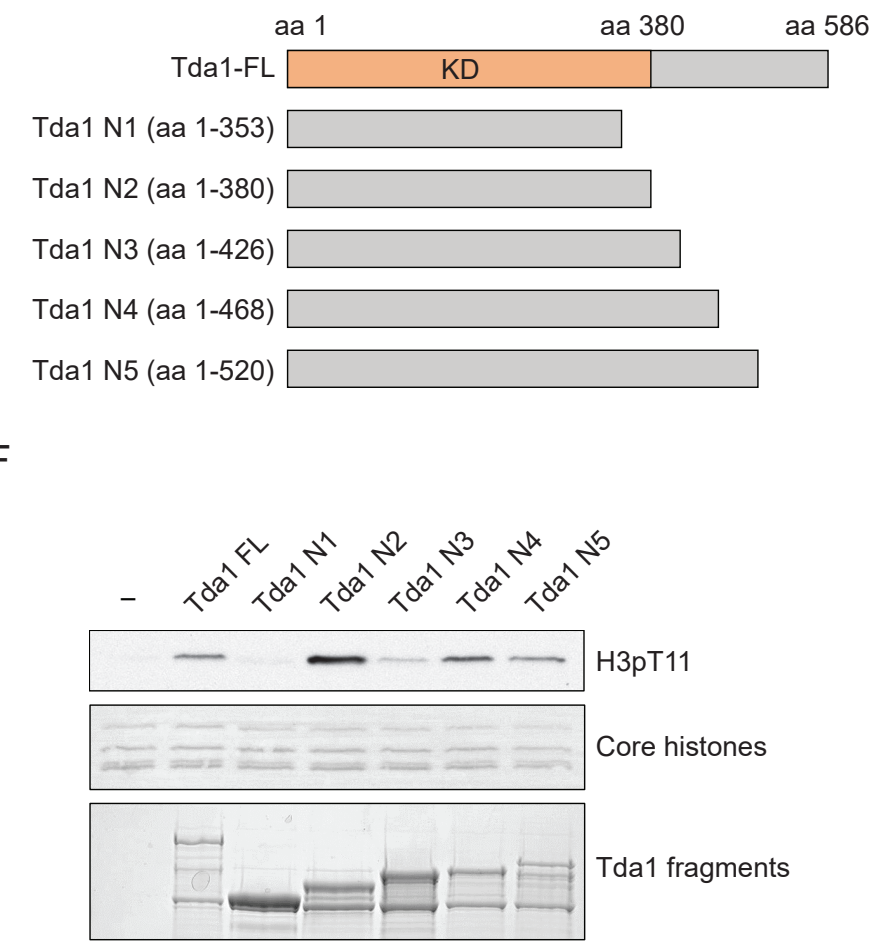


A
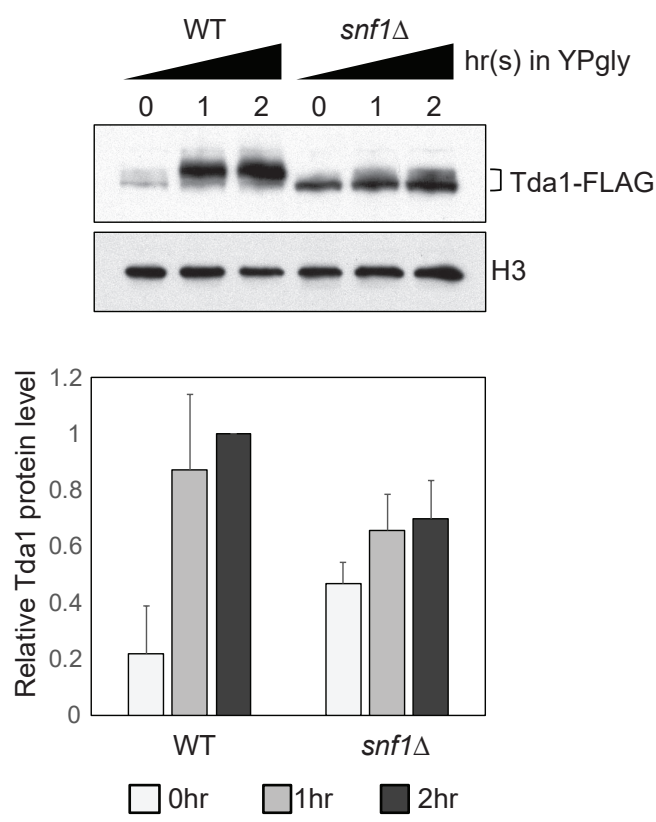

E

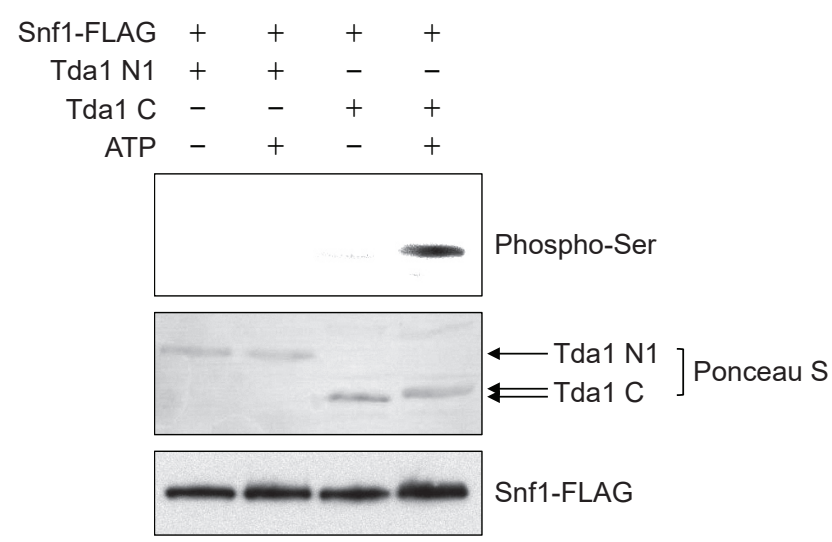

D
C
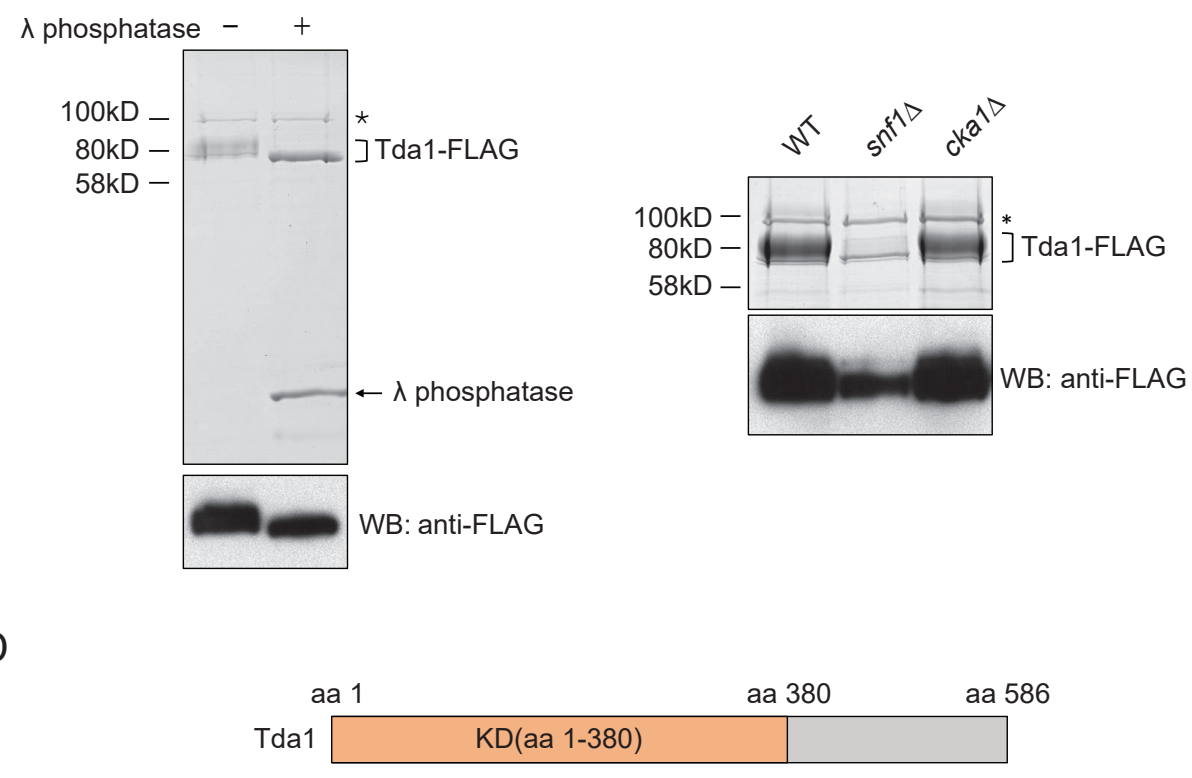

Tda1 N1(aa 1-353)

Tda1 C(aa 354-586)

F

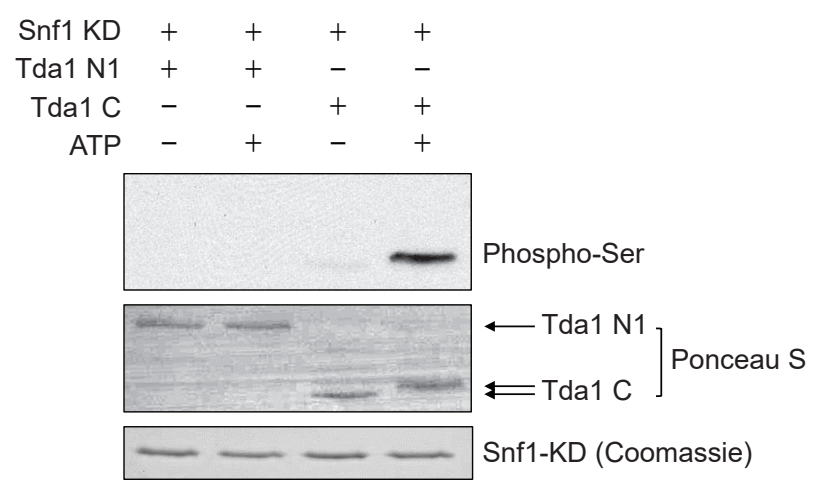


A

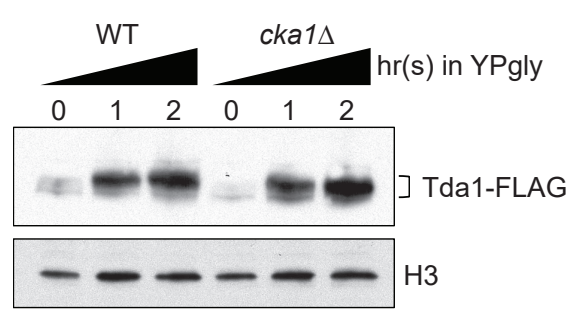

B
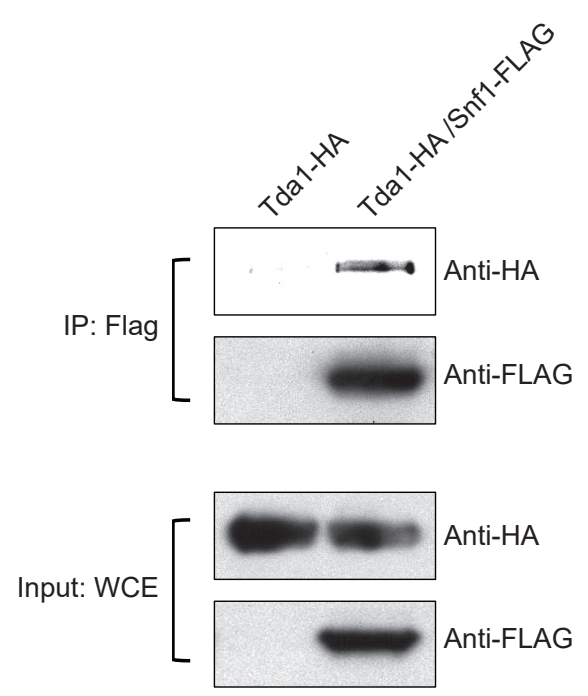

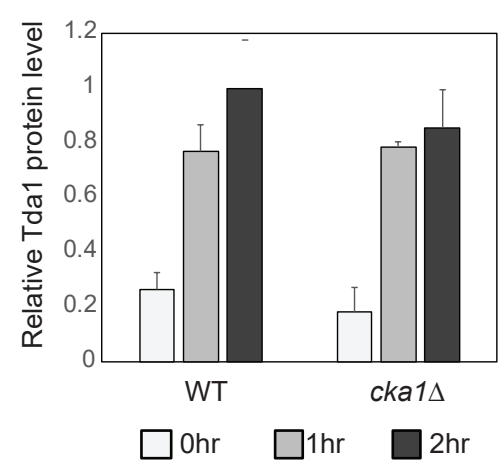

C
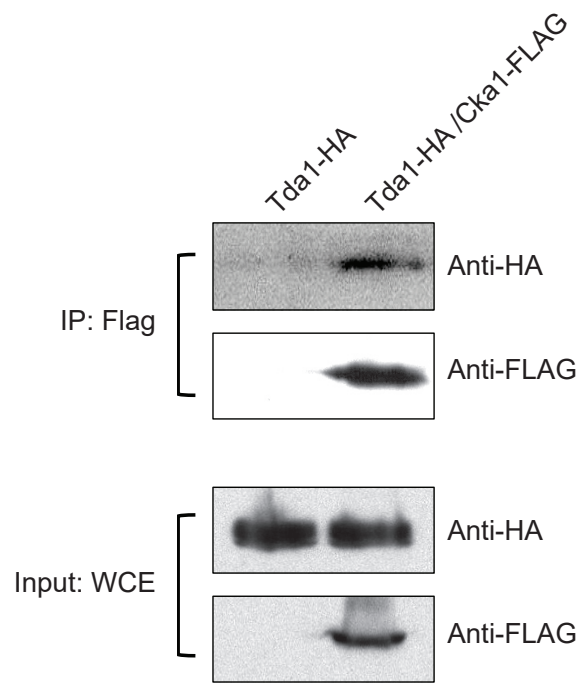

D

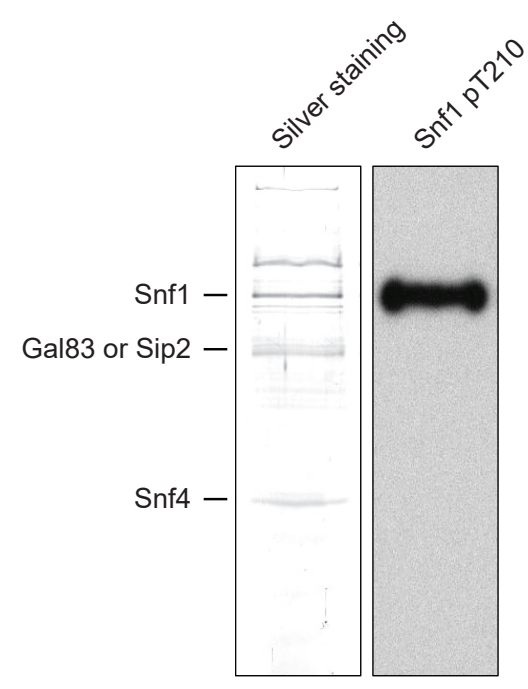


A

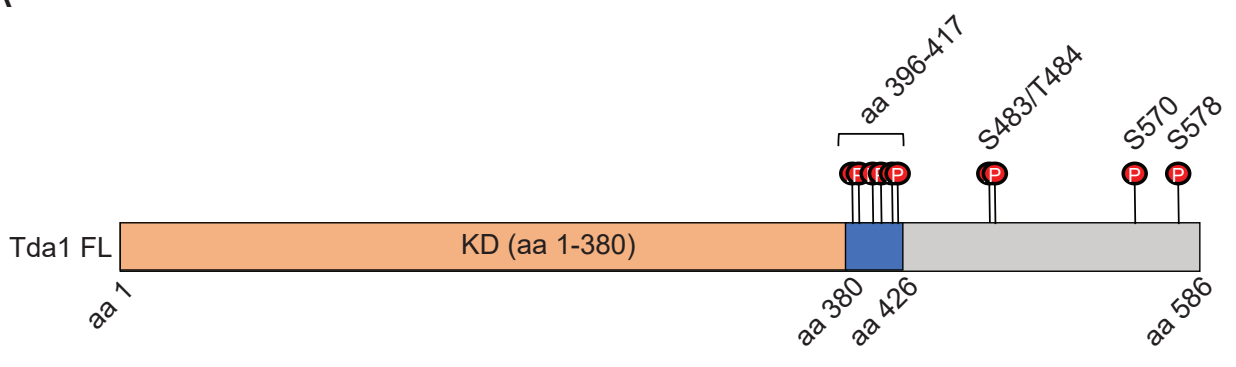

B

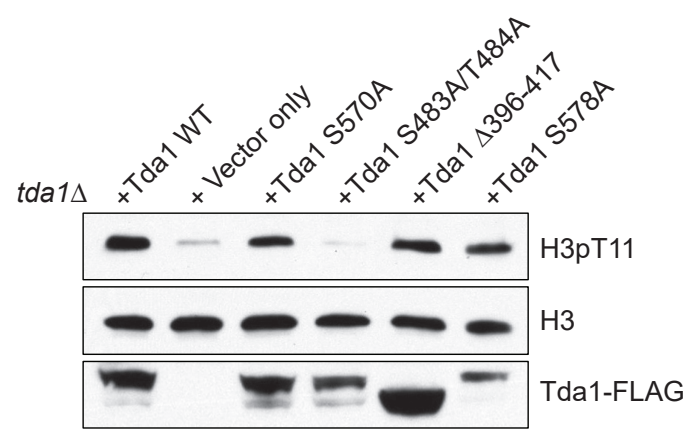

C

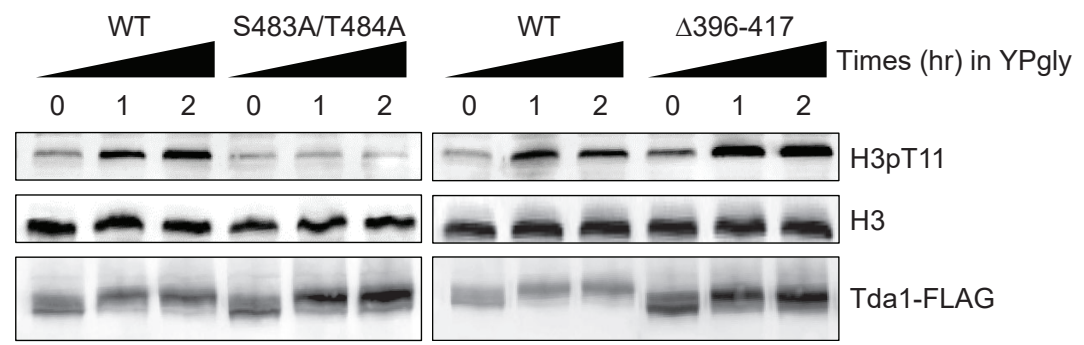

D

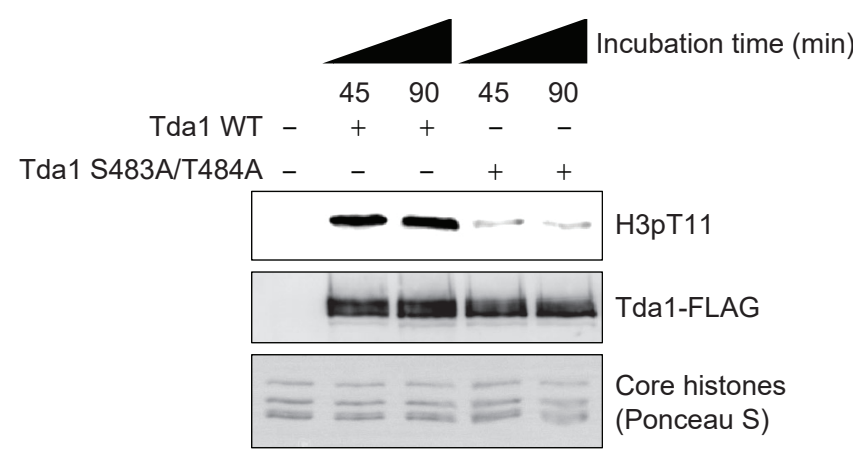


A

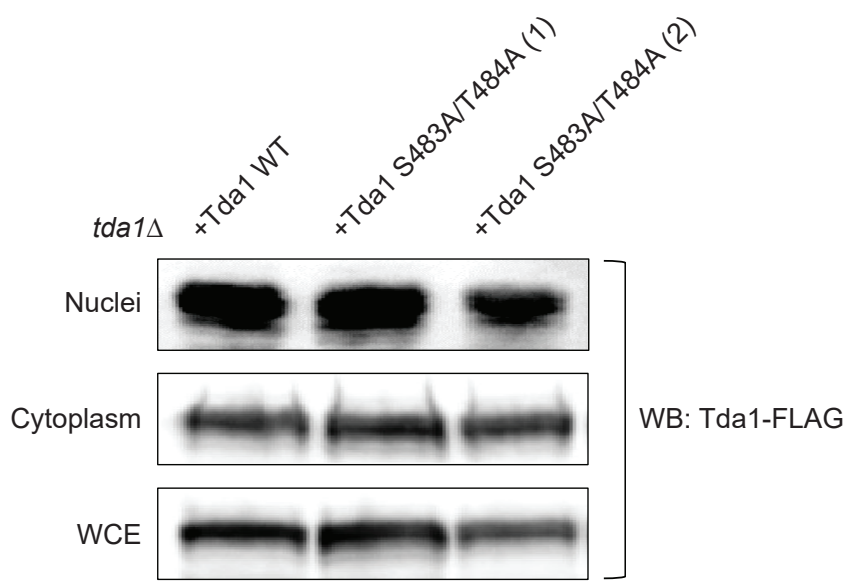

B

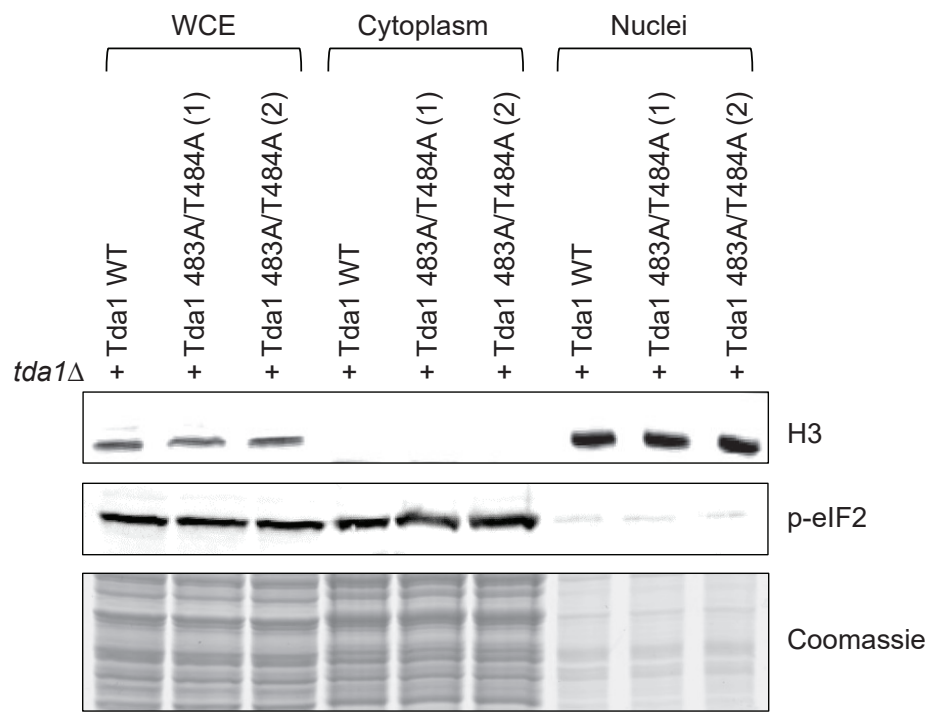


bioRxiv preprint doi: https://doi.org/10.1101/2020.11.03.367094; this version posted November 3, 2020. The copyright holder for this preprint (which was not certified by peer review) is the author/funder. All rights reserved. No reuse allowed without permission.

Figure 7

A

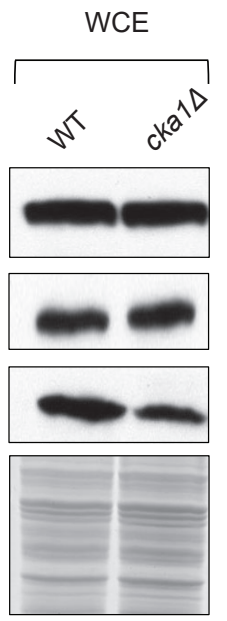

$$
\text { Cytoplasm }
$$
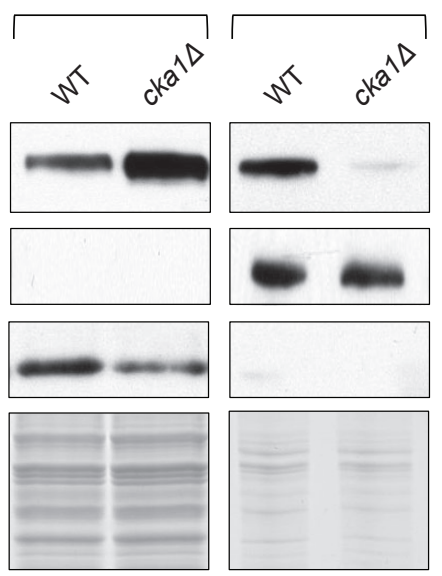

p-elF2

Coomassie

Tda1-FLAG

\section{H3}

Comassie
B

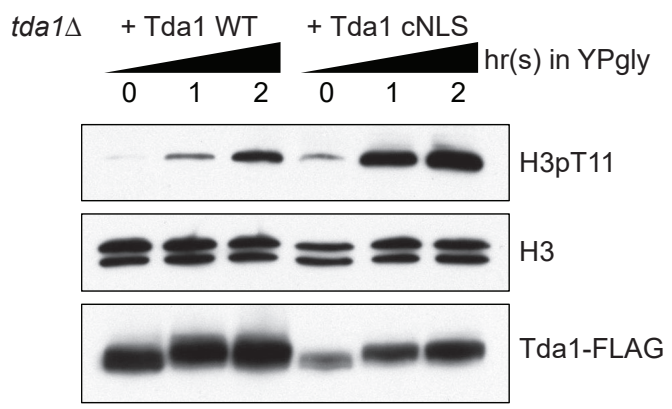

C

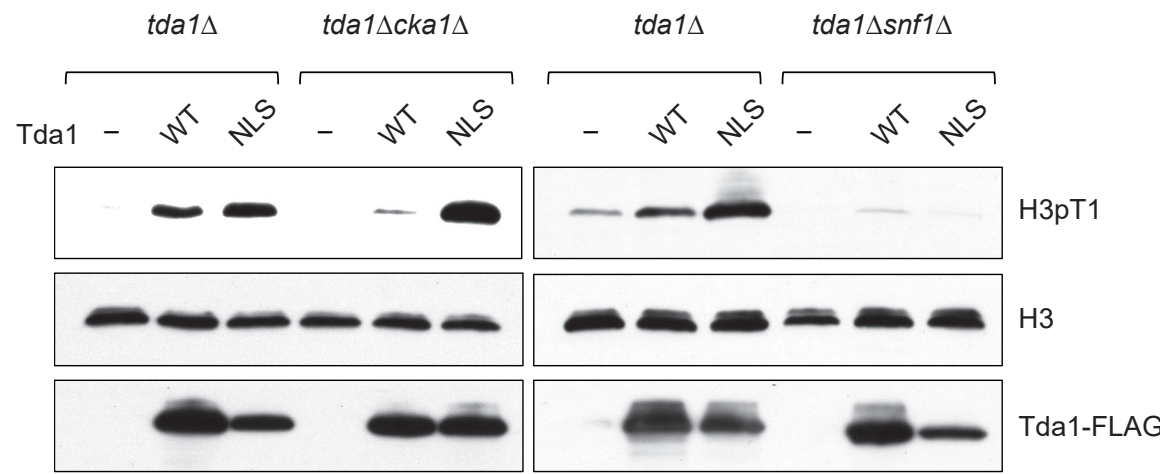

\section{C} B

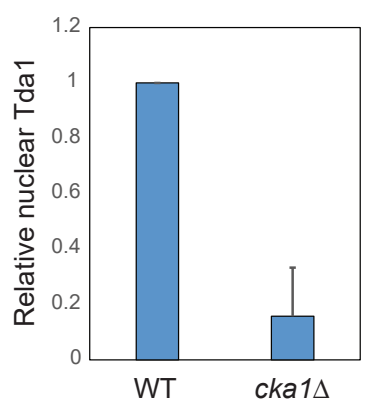


bioRxiv preprint doi: https://doi.org/10.1101/2020.11.03.367094; this version posted November 3, 2020. The copyright holder for this preprint (which was not certified by peer review) is the author/funder. All rights reserved. No reuse allowed without permission.

Figure 7 -figure supplement 1

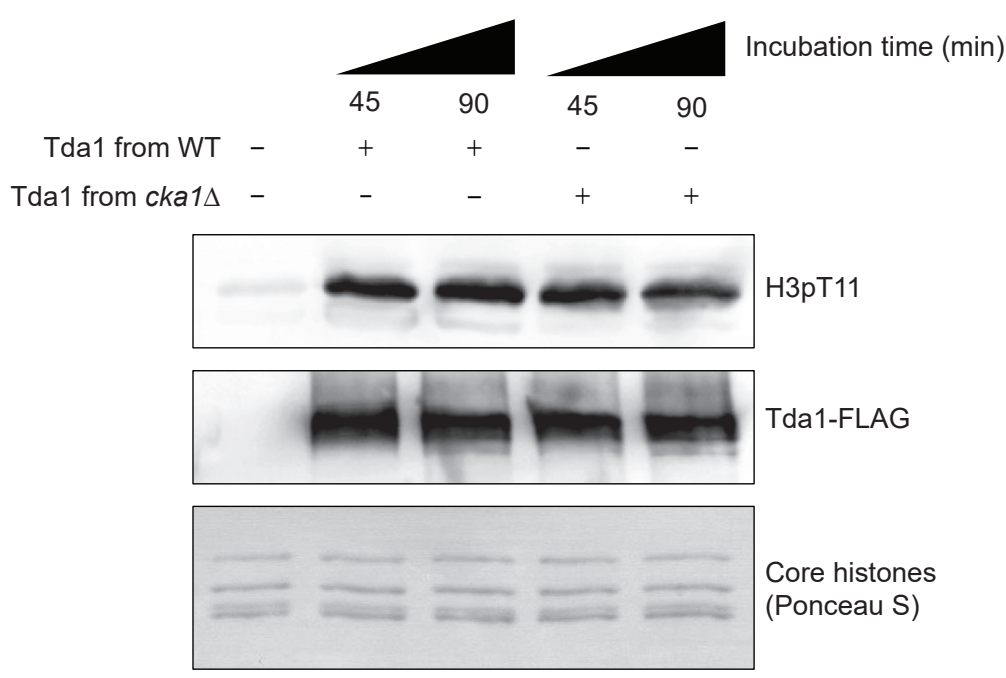

